\title{
Exotic resonances of fully-heavy tetraquarks in a lattice-QCD insipired quark model
}

\author{
Gang Yang $\odot^{1, *}$ Jialun Ping, ${ }^{2, \dagger}$ and Jorge Segovia $\oplus^{3, \$}$ \\ ${ }^{1}$ Department of Physics, Zhejiang Normal University, Jinhua 321004, China \\ ${ }^{2}$ Department of Physics, Nanjing Normal University, Nanjing 210023, China \\ ${ }^{3}$ Departamento Sistemas Físicos, Químicos y Naturales,Universidad Pablo de Olavide, \\ 41013 Sevilla, Spain
}

(Received 23 April 2021; accepted 15 June 2021; published 7 July 2021)

\begin{abstract}
Fully-heavy tetraquark states, i.e., $c c \bar{c} \bar{c}, b b \bar{b} \bar{b}, b b \bar{c} \bar{c}(c c \bar{b} \bar{b}), c b \bar{c} \bar{c}, c b \bar{b} \bar{b}$, and $c b \bar{c} \bar{b}$, are systematically investigated by means of a nonrelativistic quark model based on lattice-QCD studies of the two-body $Q \bar{Q}$ interaction, which exhibits a spin-independent Cornell potential along with a spin-spin term. The four-body problem is solved using the Gaussian expansion method; additionally, the so-called complex scaling technique is employed so that bound, resonance, and scattering states can be treated on the same footing. Moreover, a complete set of four-body configurations, including meson-meson, diquark-antidiquark, and K-type configurations, as well as their couplings, are considered for spin-parity quantum numbers $J^{P(C)}=0^{+(+)}, 1^{+( \pm)}$, and $2^{+(+)}$in the $S$-wave channel. Several narrow resonances, with two-meson strong decay widths less than $30 \mathrm{MeV}$, are found in all of the tetraquark systems studied. Particularly, the fullycharm resonances recently reported by the LHCb Collaboration, at the energy range between 6.2 and $7.2 \mathrm{GeV}$ in the di- $J / \psi$ invariant spectrum, can be well identified in our calculation. Focusing on the fullybottom tetraquark spectrum, resonances with masses between 18.9 and $19.6 \mathrm{GeV}$ are found. For the remaining charm-bottom cases, the masses are obtained within a energy region from $9.8 \mathrm{GeV}$ to $16.4 \mathrm{GeV}$. All these predicted resonances can be further examined in future experiments.
\end{abstract}

DOI: $10.1103 /$ PhysRevD.104.014006

\section{INTRODUCTION}

Many efforts have been made in the past twenty years in order to understand exotic tetra-, penta-, and even hexaquark systems, constituted mostly by heavy quarks. For example, in 2015, the hidden-charm pentaquark states $P_{c}$ (4380), $P_{c}(4312), P_{c}(4440)$, and $P_{c}(4457)$ were discovered by the LHCb collaboration [1,2], adding them to a large number of unconventional heavy mesons called jointly $\mathrm{XYZ}$ states, e.g., the $X(3872)$ discovered by the Belle Collaboration in 2003 [3], the $Y(4260)$ discovered by the BABAR Collaboration in 2005 [4], $Z_{c}(3900)$ discovered by the BESIII Collaboration in 2013 [5], etc.

The fully-heavy tetraquark states $Q Q \bar{Q} \bar{Q}(Q=c, b)$ have recently attracted much attention. In 2017, the CMS Collaboration reported a benchmark measurement of $\Upsilon(1 S)$-pair production in $p p$ collisions at $\sqrt{s}=8 \mathrm{TeV}$ [6].

\footnotetext{
*yanggang@zjnu.edu.cn

jlping@njnu.edu.cn

7jsegovia@upo.es
}

Published by the American Physical Society under the terms of the Creative Commons Attribution 4.0 International license. Further distribution of this work must maintain attribution to the author(s) and the published article's title, journal citation, and DOI. Funded by SCOAP ${ }^{3}$.
A preliminary analysis of the CMS data shows an excess at $18.4 \mathrm{GeV}$ in the $\Upsilon(1 S) \ell^{+} \ell^{-}$decay channels [7-9]. This excess, if confirmed by future experiments, may indicate a fully-bottom tetraquark state $(b b \bar{b} \bar{b})$. Besides, a significant peak at $18.2 \mathrm{GeV}$ was observed in $\mathrm{Cu}+\mathrm{Au}$ collisions at RHIC [10] but the LHCb and CMS collaborations [11,12] were not able to confirm it from the $\Upsilon(1 S) \mu^{+} \mu^{-}$invariant mass spectrum. Recently, in the di- $J / \psi$ invariant mass spectrum, a narrow peak at $6.9 \mathrm{GeV}$, a broad one between 6.2 and $6.8 \mathrm{GeV}$, and a hint for a possible structure around $7.2 \mathrm{GeV}$ were reported by the LHCb collaboration, which could indicate the existence of fully-charm tetraquarks [13]. It is then expected that more investigations on the existence of fully-heavy tetraquark states will be performed in the future in, for instance, the $\mathrm{LHCb}$ experiment at CERN.

The search of fully-heavy tetraquark states is valuable from the experimental side but also from the theoretical view point [14-16]. The debates on the existence of $Q Q \bar{Q} \bar{Q}$ $(Q=c, b)$ states were quite intense before the LHCb's findings in the di-J/ $\psi$ invariant mass spectrum. A $b b \bar{b} \bar{b}$ bound state was supported by various models [17-20], QCD sum rules studies [21,22], and a naive diffusion Monte Carlo calculation [23]. The study of its decay properties concluded that a fully-bottom tetraquark state with $J^{P}=2^{+}$is possible [24]. As for the potential $c c \bar{c} \bar{c}$ state, it was suggested that its 
mass is located between 5 and $6 \mathrm{GeV}$ through various phenomenological models [17,25-27] and the BetheSalpeter equation approach [28]. Additionally, narrow $b b \bar{b} \bar{c}$ and $b c \bar{b} \bar{c}$ tetraquark states were predicted [29,30]. On the contrary, there were counter examples on the existence of $Q Q \bar{Q} \bar{Q}$ tetraquark states. For example, it was shown that bound states of $c c \bar{c} \bar{c}$ and $b b \bar{b} \bar{b}$ systems are impossible through model investigations of Refs. [29-34] but also in preliminary lattice-QCD computations [35].

The interpretation of the newly reported structures in the di- $J / \psi$ invariant mass spectrum have been carried out by various theoretical approaches. In a nonrelativistic model with a compact diquark-antidiquark configuration, masses of the $S$-wave fully-charm tetraquark states are predicted to be between 5.96 and $6.32 \mathrm{GeV}$ [36]. In a potential model including the linear-confinement and the one-gluonexchange contributions, fully-charm tetraquark masses are predicted at $6.5 \mathrm{GeV}$ and $6.9 \mathrm{GeV}$, which can be identified with the observed structures as $S$ - and $P$-wave fully-charm tetraquarks [37]. This conclusion differs from a study with a dynamical diquark model, where the two resonances around $6.7 \mathrm{GeV}$ and $6.9 \mathrm{GeV}$ are identified with the $1 \mathrm{P}$ and $2 \mathrm{~S}$ multiplets [38]. Furthermore, the narrow and broad structures observed in the di- $J / \psi$ invariant mass spectrum are explained as radial excitations of the fully-charm state within the QCD sum rules approach [39], the string-junction picture [40], and the extended relativized quark model [41]. On the other hand, based on the perturbative QCD approach [42], it was predicted that both the narrow and broad structures prefer molecularlike configurations. On the contrary, a holography inspired model suggests a compact picture for the structure around $6.9 \mathrm{GeV}$ [43]. Concerning the quantum numbers of the di- $J / \psi$ structures, the spinparity of the $X(6900)$ resonance is suggested to be $J^{P}=2^{+}$ within the constituent quark model of Ref. [44] and the relativistic quark model of Ref. [45], while it is predicted to be $0^{+}$or $1^{+}$by an effective potential model [46] and $0^{+}$by a diffusion Monte Carlo calculation [47].

In addition, a unitary coupled-channels approach seems to describe well di-J/ $\psi$ invariant mass spectrum finding hints of a near-threshold molecular state $X(6200)$ with quantum numbers $J^{P C}=0^{++}$or $2^{++}$[48]. Meanwhile, an $a b$ initio perturbative $\mathrm{QCD}$ investigation suggested that there exists another state near the peak at $6.9 \mathrm{GeV}$ and thus the nature of $X(6900)$ can be uncovered by calculating the cross section of this undiscovered state [49]. On the other hand, strong decay properties of the fully-charm tetraquark states were investigated in Ref. [50], predicting that the broad structure is a $S$-wave state with either $J^{P C}=0^{++}$or $2^{++}$and the narrow peak can be identified as a $P$-wave state with quantum numbers $J^{P C}=0^{-+}$or $1^{-+}$. The production of the fully-charm tetraquark state at $6.9 \mathrm{GeV}$ was also studied by a $p \bar{p}$ annihilation process [51], a dynamic simulation [52], and a model-independent fragmentation mechanism [53].
In this work, we explore the possibility of having bound, resonance and scattering states of fully-heavy quark systems, viz. $Q Q \bar{Q} \bar{Q}(Q=c, b)$, with spin-parity $J^{P(C)}=0^{+(+)}, 1^{+( \pm)}$and $2^{+(+)}$in the $S$-wave channel. ${ }^{1} \mathrm{We}$ employ a nonrelativistic quark model for the two-body interaction between heavy quarks according to the LatticeQCD study of Ref. [55]. The four-body problem is solved by using the Gaussian expansion method [56], which has been demonstrated to be as accurate as a Faddeev calculation. The complete set of four-body configurations: meson-meson, diquark-antidiquark, and K-type arrangements, as well as their couplings, are considered in the calculation. Meanwhile, a coupled-channels calculation which treats the bound, resonance, and scattering states on the same footing is performed by employing the complex scaling method [57-61] according to the so-called $\mathrm{ABC}$ theorem [57,58]. This tool has been already used in previous studies of nuclear [62-65] and hadron [66-69] physics.

This manuscript is organized as follows. The theoretical framework is presented in Sec. II, including the latticeQCD inspired potential model and the general structure of the four-body wave function. Section III is devoted to the analysis and discussion of the obtained results. And we summarize our theoretical work in Sec. IV.

\section{THEORETICAL FRAMEWORK}

The kinetic motion for heavy quarks (charm and bottom) can be treated in a nonrelativistic way, and their interactions can be described via a potential model. Here, we employ a potential model inspired by the Lattice-QCD investigation of Ref. [55], viz. the interaction between a heavy quark and a heavy antiquark can be well approximated by the spinindependent Cornell potential along with a spin-spin term. For the four-body system $Q Q \bar{Q} \bar{Q}(Q=c, b)$, the Hamiltonian results to be

$$
H=\sum_{i=1}^{4}\left(M_{Q}+\frac{\hat{\mathbf{p}}_{i}^{2}}{2 M_{Q}}\right)+\sum_{j>i=1}^{4} V_{i j}\left(\mathbf{r}_{i j}\right),
$$

where $M_{Q}$ is the mass of the heavy quarks and antiquarks. The two-body interaction potential can be written as

$V_{i j}\left(\mathbf{r}_{i j}\right)=-\frac{3}{16}\left(\lambda_{i}^{a} \cdot \lambda_{j}^{a}\right)\left[-\frac{\alpha}{\left|\mathbf{r}_{i j}\right|}+\sigma\left|\mathbf{r}_{i j}\right|+\beta e^{-\gamma\left|\mathbf{r}_{i j}\right|}\left(\mathbf{s}_{i} \cdot \mathbf{s}_{j}\right)\right]$,

which includes the coulomb, linear-confining and spinspin interactions. The color-dependence of the interaction

\footnotetext{
${ }^{1}$ Note here that an exploratory analysis of this study for the $c c \bar{c} \bar{c}$ and $b b \bar{b} \bar{b}$ tetraquark systems was recently published in Ref. [54].
} 
is encoded in the $\mathrm{SU}(3)$ Gell-Mann matrices, $\lambda_{i}^{a}$ $(a=1,2, \ldots, 8)$. Since the two-body interaction depends only on the distance between the two quarks/antiquarks labeled by $i$ and $j,\left|\mathbf{r}_{i j}\right|=\left|\mathbf{r}_{i}-\mathbf{r}_{j}\right|$, the four-body problem can be factorized into a center-of-mass motion and relative motions. In the following, we subtract the center-of-mass motion and focus on the relative motions of the four-body system. The model parameters $\alpha, \beta, \gamma$, and $\sigma$ can be determined via a calculation of the mass spectrum of the $S$-wave $Q \bar{Q}$ mesons. Table I lists the values of the model parameters, which are the ones collected in Ref. [70] except the spin-spin coefficient of the charm-bottom case, $\beta_{c b}$, which is determined herein. The calculated masses of the $S$ wave $Q \bar{Q}$ mesons along with their experimental values are listed in Table II. The theoretical results are consistent with the experimental data, providing a solid ground to study possible bound and resonance states in the four-body system $Q Q \bar{Q} \bar{Q}$.

Figure 1 shows a complete set of configurations for the fully-heavy tetraquark system $Q Q \bar{Q} \bar{Q}(Q=c, b)$. Figures 1(a) and 1(b) correspond to the meson-meson structure and diquark-antidiquark one, respectively. Figures 1(c)-(f) are the so-called K-type configurations. In general, all these configurations are coupled to each other; on the other hand, it was shown [71,72] that if all

TABLE I. Model parameters.

\begin{tabular}{lcc}
\hline \hline Quark masses & $M_{c}(\mathrm{MeV})$ & 1290 \\
& $M_{b}(\mathrm{MeV})$ & 4700 \\
Coulomb & $\alpha$ & 0.4105 \\
Confinement & $\sigma\left(\mathrm{GeV}^{2}\right)$ & 0.2 \\
Spin-Spin & $\gamma(\mathrm{GeV})$ & 1.982 \\
& $\beta_{c c}(\mathrm{GeV})$ & 2.06 \\
& $\beta_{c b}(\mathrm{GeV})$ & 0.789 \\
& $\beta_{b b}(\mathrm{GeV})$ & 0.318 \\
\hline \hline
\end{tabular}

TABLE II. Theoretical and experimental values of the masses for the $S$-wave $Q \bar{Q}$ mesons (in units of $\mathrm{MeV}$ ).

\begin{tabular}{lcc}
\hline \hline State & $M_{\text {the }}$ & $M_{\text {exp }}$ \\
\hline$\eta_{c}(1 S)$ & 2968 & 2981 \\
$\eta_{c}(2 S)$ & 3655 & 3639 \\
$J / \psi(1 S)$ & 3102 & 3097 \\
$\psi(2 S)$ & 3720 & 3686 \\
$B_{c}(1 S)$ & 6275 & 6275 \\
$B_{c}(2 S)$ & 6885 & 6872 \\
$B_{c}^{*}(1 S)$ & 6349 & $\ldots$ \\
$B_{c}^{*}(2 S)$ & 6918 & $\ldots$ \\
$\eta_{b}(1 S)$ & 9401 & 9398 \\
$\eta_{b}(2 S)$ & 9961 & 9999 \\
$\Upsilon(1 S)$ & 9463 & 9460 \\
$\Upsilon(2 S)$ & 9981 & 10023 \\
\hline \hline
\end{tabular}

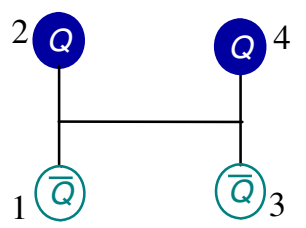

(a)

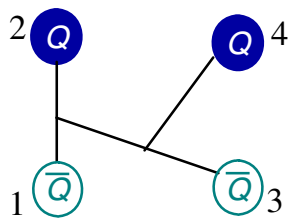

(c)

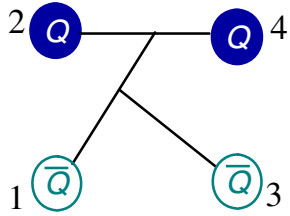

(e)

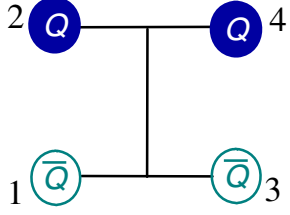

(b)

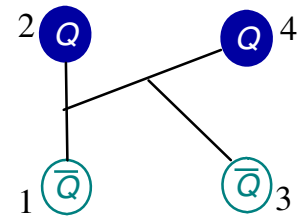

(d)

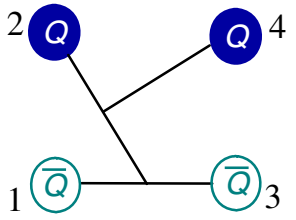

(f)
FIG. 1. All possible configurations in the fully-heavy tetraquarks. Panel (a) is the meson-meson structure, panel (b) is the diquark-antidiquark one and the other panels are of K-type. $(Q=c, b)$

possible excited states are included, it is enough to consider only the color-singlet channel. Since it is difficult to include all excited states and we are interested in the low-lying ones, it is more convenient to consider all possible configurations and the couplings among them. The price to pay following this procedure is that the overlap matrix becomes singular during the process of diagonalization because the exchange of identical particles. This can be fixed by diagonalizing the overlap matrix and drop the eigenvectors which correspond to vanishing eigenvalues. Then, a new Hamiltonian matrix can be generated using the rest of eigenvectors and the energy levels of the four-body system can be obtained from the reconstructed Hamiltonian matrix.

\section{A. Color, flavor, and spin structure}

There are four fundamental degrees of freedom for heavy quarks, i.e., color, flavor, spin, and spatial degrees of freedom.

The color-space of a tetraquark system should be spanned by 12 basis functions. In our description, they correspond to the complete configurations shown in Fig. 1 and each one gives two basis wave functions or color channels. That is to say, the meson-meson configuration, Fig. 1(a), gives two color channels, $\varphi_{1}$ and $\varphi_{2}$, corresponding to the product of two color-singlets $\left(\mathbf{1}_{\mathrm{c}} \otimes \mathbf{1}_{\mathrm{c}}\right)$ and the product of two color-octets $\left(\mathbf{8}_{\mathrm{c}} \otimes \mathbf{8}_{\mathrm{c}}\right)$. They are explicitly given by 


$$
\begin{aligned}
& \varphi_{1}=\frac{1}{3}(\overline{\mathrm{r}} \mathrm{r}+\overline{\mathrm{g} g}+\overline{\mathrm{b}} \mathrm{b}) \times(\overline{\mathrm{r}} \mathrm{r}+\overline{\mathrm{g}} \mathrm{g}+\overline{\mathrm{b}} \mathrm{b}), \\
& \varphi_{2}=\frac{\sqrt{2}}{12}(3 \bar{b} \bar{r} \bar{r} b+3 \bar{g} r \bar{r} g+3 \bar{b} g \bar{g} b+3 \bar{g} b \bar{b} g+3 \bar{r} g \bar{g} r \\
& +3 \bar{r} b \bar{b} r+2 \bar{r} r \bar{r} r+2 \bar{g} g \bar{g} g+2 \bar{b} b \bar{b} b-\bar{r} r \bar{g} g \\
& -\bar{g} g \bar{r} r-\bar{b} b \bar{g} g-\bar{b} b \bar{r} r-\bar{g} g \bar{b} b-\bar{r} r \bar{b} b) \text {. }
\end{aligned}
$$

The diquark-antidiquark configuration, Fig. 1(b), gives again two color basis, $\varphi_{3}$ and $\varphi_{4}$, corresponding to the product of a color triplet and a color antitriplet $\left(\mathbf{3}_{\mathrm{c}} \otimes \overline{\mathbf{3}}_{\mathrm{c}}\right)$ and the product of a color sextet and a color antisextet $\left(\mathbf{6}_{\mathrm{c}} \otimes \overline{\mathbf{6}}_{\mathrm{c}}\right)$. They are explicitly given by

$$
\begin{aligned}
& \varphi_{3}=\frac{\sqrt{3}}{6}(\overline{\mathrm{r}} \mathrm{r} \bar{g} g-\overline{\mathrm{g}} \mathrm{r} \overline{\mathrm{r}} \mathrm{g}+\overline{\mathrm{g} g} \mathrm{~g} \mathrm{r} \mathrm{r}-\overline{\mathrm{r}} \mathrm{g} \overline{\mathrm{g}} \mathrm{r}+\overline{\mathrm{r}} \mathrm{r} \overline{\mathrm{b}} \mathrm{b}-\overline{\mathrm{b}} \mathrm{r} \overline{\mathrm{r}} \mathrm{b} \\
& +\bar{b} b \bar{r} r-\bar{r} b \bar{b} r+\bar{g} g \bar{b} b-\bar{b} g \bar{g} b+\bar{b} b \bar{g} g-\bar{g} b \bar{b} g), \\
& \varphi_{4}=\frac{\sqrt{6}}{12}(2 \overline{\mathrm{r}} \mathrm{r} \overline{\mathrm{r}} \mathrm{r}+2 \overline{\mathrm{g} g} \mathrm{~g} \mathrm{~g} g+2 \overline{\mathrm{b}} \mathrm{b} \overline{\mathrm{b}} \mathrm{b}+\overline{\mathrm{r}} \mathrm{r} \overline{\mathrm{g} g}+\overline{\mathrm{g}} \mathrm{r} \overline{\mathrm{r} g} \\
& +\bar{g} g \bar{r} r+\bar{r} g \bar{g} r+\bar{r} r \bar{b} b+\bar{b} \bar{r} \bar{b}+\bar{b} b \bar{r} r \\
& +\overline{\mathrm{r}} \mathrm{b} \overline{\mathrm{b}} \mathrm{r}+\overline{\mathrm{g} g} \mathrm{~g} \overline{\mathrm{b}}+\overline{\mathrm{b}} \mathrm{g} \overline{\mathrm{g}} \mathrm{b}+\overline{\mathrm{b}} \mathrm{g} \overline{\mathrm{g}} \mathrm{g}+\overline{\mathrm{g}} \mathrm{b} \overline{\mathrm{b}} \mathrm{g}) .
\end{aligned}
$$

Each K-type configuration, Figs. 1(c)-(f), corresponds with two color channels. For convenience, we denote the K-type configurations from (c) to (f) in Fig. 1 as $\mathrm{K}_{1}, \mathrm{~K}_{2}, \mathrm{~K}_{3}$, and $\mathrm{K}_{4}$, respectively. The corresponding color wave functions have been constructed in Ref. [67]. The color wave functions $\varphi_{5}$ and $\varphi_{6}$, corresponding to the $\mathrm{K}_{1}$ configuration [Fig. 1(c)], are given by

$$
\varphi_{5}=\varphi_{2}, \quad \varphi_{6}=\varphi_{1} .
$$

The color wave functions $\varphi_{7}$ and $\varphi_{8}$ from the $\mathrm{K}_{2}$ configuration [Fig. 1(d)] are similar. We have

$$
\varphi_{7}=\varphi_{1}, \quad \varphi_{8}=\varphi_{2} .
$$

The color wave functions $\varphi_{9}$ and $\varphi_{10}$ from the $\mathrm{K}_{3}$ configuration [Fig. 1(e)] can be shown to be

$$
\begin{aligned}
& \varphi_{9}=\frac{1}{2 \sqrt{6}}(\overline{\mathrm{r}} \mathrm{b} \overline{\mathrm{b}} \mathrm{r}+\overline{\mathrm{r}} \mathrm{r} \overline{\mathrm{b}} \mathrm{b}+\overline{\mathrm{g}} \mathrm{b} \overline{\mathrm{b}} \mathrm{g}+\overline{\mathrm{g} g} \overline{\mathrm{b}} \mathrm{b}+\overline{\mathrm{r}} \mathrm{g} \overline{\mathrm{g}} \mathrm{r}+\overline{\mathrm{r}} \mathrm{g} \overline{\mathrm{g} g} \\
& +\bar{b} b \bar{g} g+\bar{b} g \bar{g} b+\bar{g} g \bar{g} r \mathrm{r}+\bar{g} \mathbf{r} \bar{r} g+\bar{b} b \bar{r} r+\bar{b} r \bar{r} b) \\
& +\frac{1}{\sqrt{6}}(\overline{\mathrm{r}} \mathrm{r} \overline{\mathrm{r}} \mathrm{r}+\overline{\mathrm{g} g} \overline{\mathrm{g} g} \mathrm{~g}+\overline{\mathrm{b}} \mathrm{b} \overline{\mathrm{b}} \mathrm{b}), \\
& \varphi_{10}=\frac{1}{2 \sqrt{3}}(\overline{\mathrm{r}} \mathrm{b} \overline{\mathrm{b}} \mathrm{r}-\overline{\mathrm{r}} \mathrm{r} \overline{\mathrm{b}} \mathrm{b}+\overline{\mathrm{g}} \mathrm{b} \overline{\mathrm{b}} \mathrm{g}-\overline{\mathrm{g} g} \mathrm{~b} \mathrm{~b}+\overline{\mathrm{r}} \mathrm{g} \overline{\mathrm{g}} \mathrm{r}-\overline{\mathrm{r}} \mathrm{g} \overline{\mathrm{g}} \mathrm{g} \\
& -\bar{b} b \bar{g} g+\bar{b} g \bar{g} b-\bar{g} g \bar{r} r+\bar{g} r \bar{r} g-\bar{b} b \bar{r} r+\bar{b} r \bar{r} b) .
\end{aligned}
$$

And, finally, the color wave functions $\varphi_{11}$ and $\varphi_{12}$ from the $\mathrm{K}_{4}$ configuration [Fig. 1(f)] read

$$
\varphi_{11}=\varphi_{9}, \quad \varphi_{12}=-\varphi_{10}
$$

The flavor structure of the $Q Q \bar{Q} \bar{Q}(Q=c, b)$ tetraquark system is trivial and will be neglected. The total spin quantum number can be $S=0,1$, and 2 . Since there is no spin-orbital interaction in the Hamiltonian, the third component of the total spin $\left(M_{S}\right)$ can be set to be equal to $S$ without loss of generality. The four-body spin wave functions, $\xi_{S}^{\alpha}$, for the configurations shown in Fig. 1 can be listed as

$$
\begin{aligned}
\xi_{0}^{u_{1}}= & \chi_{0,0}(1,2) \otimes \chi_{0,0}(3,4), \\
\xi_{0}^{u_{2}}= & \frac{1}{\sqrt{3}}\left[\chi_{1,1}(1,2) \otimes \chi_{1,-1}(3,4)-\chi_{1,0}(1,2) \otimes \chi_{1,0}(3,4)\right. \\
& \left.+\chi_{1,-1}(1,2) \otimes \chi_{1,1}(3,4)\right], \\
\xi_{0}^{u_{3}}= & \frac{1}{\sqrt{3}}\left[\chi_{1,1}(1,2) \otimes\left|\downarrow_{3} \downarrow_{4}\right\rangle+\chi_{1,-1}(1,2) \otimes\left|\uparrow_{3} \uparrow_{4}\right\rangle\right. \\
& \left.-\chi_{1,0}(1,2) \otimes \chi_{0,0}(3,4)\right], \\
\xi_{0}^{u_{4}}= & \frac{1}{\sqrt{2}} \chi_{0,0}(1,2) \otimes\left(\left|\uparrow_{3} \downarrow_{4}\right\rangle-\left|\downarrow_{3} \uparrow_{4}\right\rangle\right), \\
\xi_{1}^{v_{1}}= & \chi_{0,0}(1,2) \otimes \chi_{1,1}(3,4), \\
\xi_{1}^{v_{2}}= & \chi_{1,1}(1,2) \otimes \chi_{0,0}(3,4), \\
\xi_{1}^{v_{3}}= & \frac{1}{\sqrt{2}}\left[\chi_{1,1}(1,2) \otimes \chi_{1,0}(3,4)-\chi_{1,0}(1,2) \otimes \chi_{1,1}(3,4)\right], \\
\xi_{1}^{v_{4}}= & \sqrt{\frac{3}{4}} \chi_{1,1}(1,2) \otimes\left|\uparrow_{3} \downarrow_{4}\right\rangle-\sqrt{\frac{1}{12}} \chi_{1,1}(1,2) \otimes\left|\downarrow_{3} \uparrow_{4}\right\rangle \\
& -\sqrt{\frac{1}{6}} \chi_{1,0}(1,2) \otimes\left|\uparrow_{3} \uparrow_{4}\right\rangle, \\
\xi_{1}^{v_{5}}= & \sqrt{\frac{2}{3}} \chi_{1,1}(1,2) \otimes\left|\uparrow_{3} \downarrow_{4}\right\rangle-\sqrt{\frac{1}{3}} \chi_{1,0}(1,2) \otimes\left|\uparrow_{3} \uparrow_{4}\right\rangle, \\
\xi_{1}^{v_{6}}= & \chi_{0,0}(1,2) \otimes\left|\uparrow_{3} \uparrow_{4}\right\rangle, \\
\xi_{2}= & \chi_{1,1}(1,2) \otimes \chi_{1,1}(3,4) .
\end{aligned}
$$

The indices $u_{1}, \ldots, u_{4}, v_{1}, \ldots, v_{6}$, and $w_{1}$ number the spin channels corresponding to the configurations shown in Fig. 1. Their specific values are listed in Table III. These spin wave functions are obtained by considering the coupling of sub-clusters with the $\mathrm{SU}(2)$ algebra. The necessary bases are given by

$$
\begin{aligned}
& \chi_{1,1}(i, j)=\left|\uparrow_{i} \uparrow_{j}\right\rangle, \quad \quad \chi_{1,-1}(i, j)=\left|\downarrow_{i} \downarrow_{j}\right\rangle, \\
& \chi_{1,0}(i, j)=\frac{1}{\sqrt{2}}\left(\left|\uparrow_{i} \downarrow_{j}\right\rangle+\left|\downarrow_{i} \uparrow_{j}\right\rangle\right), \\
& \chi_{0,0}(i, j)=\frac{1}{\sqrt{2}}\left(\left|\uparrow_{i} \downarrow_{j}\right\rangle-\left|\downarrow_{i} \uparrow_{j}\right\rangle\right) .
\end{aligned}
$$


TABLE III. Indices of the spin wave functions in Eq. (9). Their specific numbers are listed in the columns corresponding to the configurations shown in Fig. 1.

\begin{tabular}{lcccccc}
\hline \hline & Dimeson & Diquark-antidiquark & $K_{1}$ & $K_{2}$ & $K_{3}$ & $K_{4}$ \\
\hline$u_{1}$ & 1 & 3 & & & & \\
$u_{2}$ & 2 & 4 & & & & \\
$u_{3}$ & & & 5 & 7 & 9 & 11 \\
$u_{4}$ & & 4 & 6 & 10 & 12 \\
$v_{1}$ & 1 & 5 & & & & \\
$v_{2}$ & 2 & 6 & & & & \\
$v_{3}$ & 3 & & 7 & 10 & 13 & 16 \\
$v_{4}$ & & & 8 & 11 & 14 & 17 \\
$v_{5}$ & & & 9 & 12 & 15 & 18 \\
$v_{6}$ & & & & &
\end{tabular}

\section{B. Computational method}

We solve the four-body problem by means of an exact and high-efficiency numerical approach, the Gaussian expansion method [56]. Three relative orbital motions of the four-body system are all expanded by using the Gaussian bases,

$$
\phi_{n l m}(\mathbf{r})=N_{n l}|\mathbf{r}|^{l} e^{-\nu_{n}|\mathbf{r}|^{2}} Y_{l m}(\hat{\mathbf{r}})
$$

where the widths $\nu_{n}$ are taken as the sizes of the geometric progression $^{2}$ and the normalization factor $N_{n l}$ is not shown explicitly. The general spatial wave function of the fourbody system can be formally expressed as

$$
\psi_{L M_{L}}=\left[\left[\phi_{n_{1} l_{1} m_{1}}(\boldsymbol{\rho}) \phi_{n_{2} l_{2} m_{2}}(\lambda)\right]_{l m} \phi_{n_{3} l_{3} m_{3}}(\mathbf{R})\right]_{L M_{L}},
$$

where $\boldsymbol{\rho}, \boldsymbol{\lambda}$, and $\mathbf{R}$ are the internal Jacobi coordinates for the four-body configurations in Fig. 1. For the meson-meson configuration, Fig. 1(a), they are given by

$$
\begin{gathered}
\boldsymbol{\rho}=\mathbf{r}_{1}-\mathbf{r}_{2}, \quad \lambda=\mathbf{r}_{3}-\mathbf{r}_{4}, \\
\mathbf{R}=\frac{m_{1} \mathbf{r}_{1}+m_{2} \mathbf{r}_{2}}{m_{1}+m_{2}}-\frac{m_{3} \mathbf{r}_{3}+m_{4} \mathbf{r}_{4}}{m_{3}+m_{4}} .
\end{gathered}
$$

For the diquark-antdiquark configuration, Fig. 1(b), they read

$$
\begin{gathered}
\boldsymbol{\rho}=\mathbf{r}_{1}-\mathbf{r}_{3}, \quad \lambda=\mathbf{r}_{2}-\mathbf{r}_{4}, \\
\mathbf{R}=\frac{m_{1} \mathbf{r}_{1}+m_{3} \mathbf{r}_{3}}{m_{1}+m_{3}}-\frac{m_{2} \mathbf{r}_{2}+m_{4} \mathbf{r}_{4}}{m_{2}+m_{4}} .
\end{gathered}
$$

For the K-type configurations in Figs. 1(c)-(f), we have

\footnotetext{
${ }^{2}$ The details of the Gaussian parameters can be found in Refs. [73].
}

$$
\begin{aligned}
\boldsymbol{\rho} & =\mathbf{r}_{i}-\mathbf{r}_{j}, \\
\lambda & =\mathbf{r}_{k}-\frac{m_{i} \mathbf{r}_{i}+m_{j} \mathbf{r}_{j}}{m_{i}+m_{j}}, \\
\mathbf{R} & =\mathbf{r}_{l}-\frac{m_{i} \mathbf{r}_{i}+m_{j} \mathbf{r}_{j}+m_{k} \mathbf{r}_{k}}{m_{i}+m_{j}+m_{k}},
\end{aligned}
$$

where the indices $i, j, k, l$ should be properly assigned according to different K-type configurations. Furthermore, with these coordinates the center-of-mass motion can be completely eliminated.

The complete basis set of the four-body wave functions that fulfill the Pauli principle can be expressed as

$$
\Psi_{J M_{J}, \alpha \beta}=\mathcal{A}\left[\left(\psi_{L M_{L}} \otimes \xi_{S M_{S}}^{\alpha}\right)_{J M_{J}} \otimes \varphi_{\beta}\right],
$$

where $J$ is the quantum number of the total angular momentum. Here the antisymmetrizing operator $\mathcal{A}$ is added considering the exchange of identical particles. Particularly, for the fully-charm, -bottom and $\bar{c} b \bar{c} b$ tetraquark states, it is explicitly given by

$$
\mathcal{A}=1-(13)-(24)+(13)(24) \text {. }
$$

Meanwhile, for the $\bar{c} c \bar{c} b$ and $\bar{b} b \bar{b} c$ tetraquark states, it reads as

$$
\mathcal{A}=1-(13) \text {, }
$$

and $\mathcal{A}=1$ for the $\bar{c} c \bar{b} b$ case. This antisymmetrizing procedure is necessary since the basis set is constructed from sub-clusters, i.e., meson-meson, diquark-antidiquark, and K-type structures. The Hamiltonian matrix can be constructed using this complete basis set of the four-body wave functions.

We employ the complex scaling method (CSM) [57-63] according to the $\mathrm{ABC}$ theorem $[57,58]$ in order to search for possible resonant states of the fully-heavy tetraquark system. Using the CSM, the resonance energy (its position and width) is obtained as a stable eigenvalue of the complex scaled Schrödinger equation:

$$
[H(\theta)-E(\theta)] \Psi_{J M, \alpha \beta}(\theta)=0,
$$

where $H(\theta)$ is obtained from the original Hamiltonian by transforming the four-body Jacobi coordinates with respect to a common complex scaling angle $\theta$ :

$|\boldsymbol{\rho}| \rightarrow|\boldsymbol{\rho}| e^{i \theta}, \quad|\lambda| \rightarrow|\lambda| e^{i \theta}, \quad|\mathbf{R}| \rightarrow|\mathbf{R}| e^{i \theta}$.

According to the $\mathrm{ABC}$ theorem $[57,58]$, the eigenvalues of Eq. (19) can be separated into three groups:

(i) A bound state corresponds to a pole on the real axis whose energy is smaller than the meson-meson threshold for a specific $J^{P(C)}$ state. It remains 
unchanged under the complex scaling transformation and such energy is identified as the mass of the four-body bound state.

(ii) The discretized continuum states, associated with the cuts, are rotated downward by an angle of $2 \theta$ with respect the real axis.

(iii) A resonant state corresponds to a complex pole, $E_{\text {res }}$, that is independent of the angle $\theta$. Moreover, it is isolated from the discretized continuum spectrum, lying along the $2 \theta$-rotated line when the relation $\tan 2 \theta>-\operatorname{Im}\left(E_{\text {res }}\right) / \operatorname{Re}\left(E_{\text {res }}\right)$ is satisfied. The mass $M$ and width $\Gamma$ of the four-body resonance are given by

$$
M=\operatorname{Re}\left(E_{\text {res }}\right), \quad \Gamma=-2 \operatorname{Im}\left(E_{\text {res }}\right) .
$$

\section{RESULTS AND DISCUSSION}

We present herein our results for the low-lying $S$-wave states $(L=0$ and hence $J=S)$ of the fully-heavy tetraquark systems $Q Q \bar{Q} \bar{Q}(Q=c, b)$. They are organized as $c c \bar{c} \bar{c}, b b \bar{b} \bar{b}, c b \bar{c} \bar{c}, c b \bar{b} \bar{b}, b b \bar{c} \bar{c}$ and $c b \bar{c} \bar{b}$ tetraquark states. Since the wave function of the tetraquark system is constructed at the fundamental quark level, some forbidden dimeson channels at hadron level, such as the di- $J / \psi$ structure $J / \psi J / \psi$ in the $1^{+(-)}$state, are included; however, they will exhibit self-consistently scattering nature in our numerical calculations. These forbidden dimeson channels, interpreted as thresholds, are actually useful for us to identify possible tetraquark states.

\section{A. Fully-charm system $c c \bar{c} \bar{c}$}

We find no bound state for all $S$-wave channels $J^{P(C)}=0^{+(+)}, 1^{+(-)}$, and $2^{+(+)}$of fully-charm system $c c \bar{c} \bar{c}$. However, using the CSM, we identify several narrow resonances. Table IV summarizes our findings, the results are discussed in the following.

\section{The $J^{P(C)}=0^{+(+)}$channel}

To seek for possible bound states of the fully-charm tetraquark system, we first consider the real case $\left(\theta=0^{\circ}\right)$, shown in Table V. Therein, the lowest energies of all channels from a fully-uncoupled calculation (without considering any channel-channel coupling), the lowest energies of all structures from a partially-coupled calculation (considering only the couplings among the channels belonging to each structure), and the lowest energy from a fully-coupled calculation (considering all channel-channel couplings), are listed. The energy (mass) spectrum from the fully-coupled calculation is also shown in Fig. 2 (green circles on the real axis).

The meson-meson configuration includes two channels, $\eta_{c} \eta_{c}$ and $J / \psi J / \psi$, and the diquark-antidiquark configuration also includes two channels, $[c c]^{\mathbf{3}_{\mathrm{c}}}[\bar{c} \bar{c}]^{\overline{\mathrm{s}}_{\mathrm{c}}}$ and
TABLE IV. Predicted resonances of the fully-charm tetraquark system from the complete coupled-channel calculation with CSM. Their masses and widths are summarized in the third and fourth column, respectively, in units of $\mathrm{MeV}$.

\begin{tabular}{llcc}
\hline \hline$J^{P(C)}$ & \multicolumn{1}{c}{ Resonance } & Mass & Width \\
\hline $0^{+(+)}$ & $\eta_{c}(1 S) \eta_{c}(2 S)$ & 6640 & 0.66 \\
& $\eta_{c}(1 S) \eta_{c}(2 S)$ & 6762 & 1.14 \\
& $J / \psi(1 S) \psi(2 S)$ & 6875 & 3.74 \\
& $J / \psi(1 S) \psi(2 S)$ & 6987 & 4.00 \\
& $J / \psi(1 S) \psi(2 S)$ & 7195 & 8.00 \\
$1^{+(-)}$ & $J / \psi(1 S) J / \psi(1 S)$ & 6274 & 0.06 \\
& $J / \psi(1 S) J / \psi(1 S)$ & 6653 & 1.20 \\
& $\eta_{c}(2 S) J / \psi(1 S)$ & 6885 & 4.10 \\
$2^{+(+)}$ & $J / \psi(1 S) \psi(2 S)$ & 7007 & 1.02 \\
\hline \hline
\end{tabular}

TABLE V. Calculation of the $J^{P(C)}=0^{+(+)}$state of the fullycharm system in the real case $\left(\theta=0^{\circ}\right)$. The first and second columns show all possible structures and channels. They are obtained by the necessary bases in spin and color degrees of freedom summarized in the third column. Note also that, in the first column, when corresponding, the theoretical value of the noninteracting meson-meson threshold appears in parentheses. The lowest energies of all channels without considering any channel-channel coupling are listed in the fourth column. The last column shows the lowest energies in a calculation taking into account the couplings among the channels belonging to each structure (configuration). The last row gives the lowest energy from the complete coupled-channel calculation. All results are in units of MeV.

\begin{tabular}{|c|c|c|c|c|}
\hline Structure & Channel & $\xi_{0}^{\alpha} ; \varphi_{\beta}[\alpha ; \beta]$ & $M_{\text {uncoupled }}$ & $M_{\text {coupled }}$ \\
\hline$\eta_{c} \eta_{c}(5936)$ & 1 & {$[1 ; 1]$} & 5936 & \\
\hline$J / \psi J / \psi(6204)$ & 2 & {$[2 ; 1]$} & 6204 & 5936 \\
\hline$\eta_{c}^{8} \eta_{c}^{8}$ & 3 & {$[1 ; 2]$} & 6403 & \\
\hline$J / \psi^{8} J / \psi^{8}$ & 4 & {$[2 ; 2]$} & 6346 & 6268 \\
\hline$[c c]^{\mathbf{6}_{\mathrm{c}}}[\bar{c} \bar{c}]^{\overline{\mathbf{6}}_{\mathrm{c}}}$ & 5 & {$[3 ; 4]$} & 6404 & \\
\hline$[c c]^{\mathbf{3}_{\mathrm{c}}}[\bar{c} \bar{c}]^{\mathbf{3}_{\mathrm{c}}}$ & 6 & {$[4 ; 3]$} & 6421 & 6343 \\
\hline \multirow[t]{4}{*}{$\mathrm{K}_{1}$} & 7 & {$[5 ; 5]$} & 6349 & \\
\hline & 8 & {$[5 ; 6]$} & 6341 & \\
\hline & 9 & {$[6 ; 5]$} & 6416 & \\
\hline & 10 & {$[6 ; 6]$} & 6160 & 6150 \\
\hline \multirow[t]{4}{*}{$\mathrm{K}_{2}$} & 11 & {$[7 ; 7]$} & 6341 & \\
\hline & 12 & {$[7 ; 8]$} & 6349 & \\
\hline & 13 & {$[8 ; 7]$} & 6160 & \\
\hline & 14 & {$[8 ; 8]$} & 6416 & 6150 \\
\hline \multirow[t]{2}{*}{$\mathrm{K}_{3}$} & 15 & {$[9 ; 10]$} & 6420 & \\
\hline & 16 & {$[10 ; 9]$} & 6402 & 6340 \\
\hline \multirow[t]{2}{*}{$\mathrm{K}_{4}$} & 17 & {$[11 ; 12]$} & 6420 & \\
\hline & 18 & {$[12 ; 11]$} & 6402 & 6340 \\
\hline \multicolumn{4}{|c|}{ Lowest energy of the fully-coupled result: } & 5936 \\
\hline
\end{tabular}




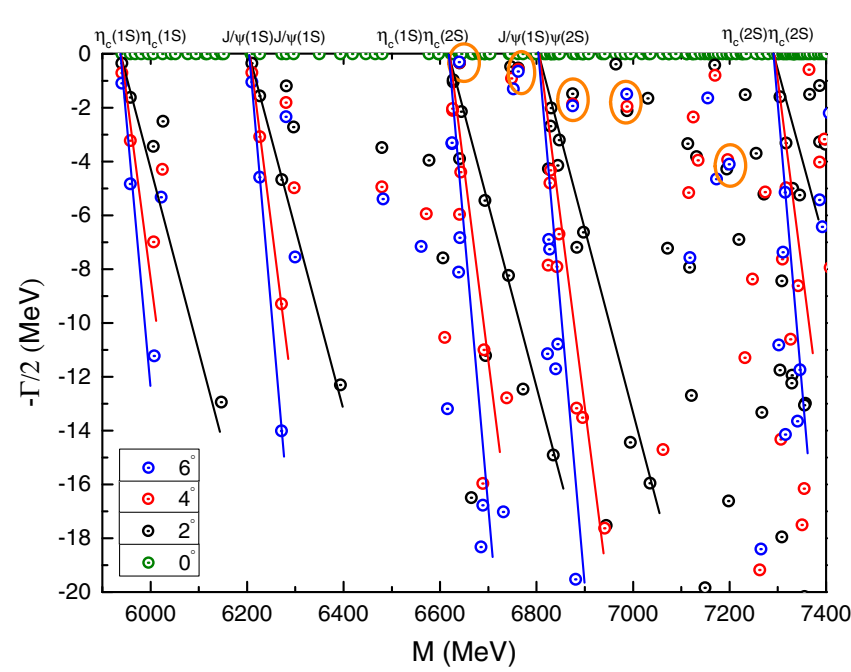

FIG. 2. Complex energy spectrum of the fully-charm tetraquark system with $J^{P(C)}=0^{+(+)}$from the complete coupled-channel calculation with CSM. The parameter $\theta$ varies from $0^{\circ}$ to $6^{\circ}$. Remember here that, according to the $\mathrm{ABC}$ theorem, (i) a bound state corresponds to a pole on the real axis whose energy is smaller than the meson-meson threshold and remains unchanged under the complex scaling transformation; (ii) the discretized continuum states are rotated downward by an angle of $2 \theta$ with respect the real axis; and (iii) a resonant state corresponds to a complex pole, that is independent of the angle $\theta$, circled in orange.

$[c c]^{\mathbf{6}_{c}}[\bar{c} \bar{c}]^{\overline{\boldsymbol{6}}_{\mathrm{c}}}$. In Table $\mathrm{V}$ we notice that the theoretical values of the dimeson threshold, $5936 \mathrm{MeV}$ for $\eta_{c} \eta_{c}$ and $6204 \mathrm{MeV}$ for $J / \psi J / \psi$, are quite comparable with the experimental values $5962 \mathrm{MeV}$ and $6194 \mathrm{MeV}$, respectively. Then in the fully-uncoupled calculation, bound state is unavailable in these channels. Particularly, the masses of the hidden-color channels $\eta_{c} \eta_{c}$ and $J / \psi J / \psi$ are both around $6.4 \mathrm{GeV}$, which is also very close to those of the diquark-antidiquark channels. Meanwhile, the masses of the K-type channels, which are all above the dimeson thresholds, generally range from 6.2 to $6.4 \mathrm{GeV}$.

In the partially-coupled calculations, which are performed for each kind of configuration, the calculated masses are still all above the lowest threshold value, $5936 \mathrm{MeV}$ for $\eta_{c} \eta_{c}$. From Table $\mathrm{V}$ we find that, the masses of the excited states for the diquark-antidiquark and K-type configurations range from 6.15 to $6.35 \mathrm{GeV}$. Since we are treating a tetraquark system in which all quarks and antiquarks have the same flavor, no interaction in the Hamiltonian (1) can distinguish between the configurations $\mathrm{K}_{1}$ and $\mathrm{K}_{2}$, as well as between $\mathrm{K}_{3}$ and $\mathrm{K}_{4}$; Thus, the results are degenerate and this is also true for other $J^{P(C)}$ states.

Finally, in the fully-coupled calculation, the lowest mass of the spectrum is $5936 \mathrm{MeV}$, which is still slightly above the $\eta_{c} \eta_{c}$ threshold. Therefore, we can conclude that no bound state exists with $J^{P(C)}=0^{+(+)}$. However, as it will be shown next, several resonances are found in the complex-range and complete coupled-channel calculation.
Figure 2 presents the complex energy spectrum with the angle $\theta$ ranging from $0^{\circ}$ to $6^{\circ}$. In the mass range of 5.9-7.4 GeV, there are five meson-meson thresholds, $\quad \eta_{c}(1 S) \eta_{c}(1 S), \quad J / \psi(1 S) J / \psi(1 S), \quad \eta_{c}(1 S) \eta_{c}(2 S)$, $J / \psi(1 S) \psi(2 S)$, and $\eta_{c}(2 S) \eta_{c}(2 S)$. The calculated complex eigenenergies are generally aligned along the threshold lines, which are rotated downward making an angle of $2 \theta$ with the real axis, indicating the nature of the scattering states. Being cautious due to the calculation noise in the highly excited region, we can identify five fixed poles that are independent of $\theta$, as circled in Fig. 2. Apparently, they can be identified as the resonance states of the fully-charm system $\operatorname{cc} \bar{c} \bar{c}$.

The first two poles, with masses and widths $(6640 \mathrm{MeV}$, $0.66 \mathrm{MeV})$ and $(6762 \mathrm{MeV}, 1.14 \mathrm{MeV})$, are just above the $\eta_{c}(1 S) \eta_{c}(2 S)$ threshold lines. Therefore, they can be identified as di- $\eta_{c}$ resonances, with a radial excitation. Similarly, the other three poles seems to be resonances of the type $J / \psi(1 S) \psi(2 S)$, and their masses and widths are (6875 MeV, $3.74 \mathrm{MeV}),(6987 \mathrm{MeV}, 4.00 \mathrm{MeV})$ and (7195 MeV, $8.00 \mathrm{MeV}$ ), respectively.

It is worth highlighting that the obtained $J / \psi(1 S) \psi(2 S)$ resonances with masses $6875 \mathrm{MeV}$ and $6987 \mathrm{MeV}$ are both quite close to the narrow structure around $6.9 \mathrm{GeV}$ claimed by the LHCb collaboration [13] and hence they could be candidates for this. Meanwhile, the remaining $\eta_{c}(1 S) \eta_{c}(2 S)$ and $J / \psi(1 S) \psi(2 S)$ resonances are consistent with the broad structure between 6.2 and $6.8 \mathrm{GeV}$, and the possible peak shown around $7.2 \mathrm{GeV}$ [13]. Their predicted small widths indicate that these resonances are stable against two-meson strong decays, indicating that they could be confirmed in future experiments.

\section{The $J^{P(C)}=1^{+(-)}$channel}

There are 23 channels in this case as listed in Table VI. We have four meson-meson channels, including the colorsinglet and hidden-color states of $\eta_{c} J / \psi$ and $J / \psi J / \psi$, one diquark-antidiquark channel $[c c]^{\mathbf{6}_{\mathrm{c}}}[\bar{c} \bar{c}]^{\bar{b}_{\mathrm{c}}}$, and $18 \mathrm{~K}$-type ones. A bound state is still unavailable in each singlechannel calculation. In particular, the theoretical masses for the two lowest dimeson states, $\eta_{c} J / \psi$ and $J / \psi J / \psi$, in the color-singlet channel, are $6070 \mathrm{MeV}$ and $6204 \mathrm{MeV}$, respectively. Moreover, the coupled-channel effect is too weak to acquire binding energy within these two channels. In the partially-coupled calculation, the masses of $\mathrm{K}_{1}$ and $\mathrm{K}_{2}$ configurations are remarkably low due to the strong coupled-channel effect. However, these masses $(6271 \mathrm{MeV})$ are still above the theoretical thresholds of $\eta_{c} J / \psi$ and $J / \psi J / \psi$. In the fully-coupled calculation, the lowest mass of the spectrum is $6070 \mathrm{MeV}$, which is still slightly above the $\eta_{c} J / \psi$ threshold. Therefore, we conclude that no bound state exists with $J^{P(C)}=1^{+(-)}$.

Within the CSM, the complete coupled-channel calculation for $J^{P(C)}=1^{+(-)} c c \bar{c} \bar{c}$ tetraquark system has been performed and the complex energy spectrum is shown in 
TABLE VI. Calculation of the $J^{P(C)}=1^{+(-)}$state of the fullycharm system in the real case $\left(\theta=0^{\circ}\right)$. The first and second columns show all possible structures and channels. They are obtained by the necessary bases in spin and color degrees of freedom summarized in the third column. Note also that, in the first column, when corresponding, the theoretical value of the noninteracting meson-meson threshold appears in parentheses. The lowest energies of all channels without considering any channel-channel coupling are listed in the fourth column. The last column shows the lowest energies in a calculation taking into account the couplings among the channels belonging to each structure (configuration). The last row gives the lowest energy from the complete coupled-channel calculation. All results are in units of $\mathrm{MeV}$.

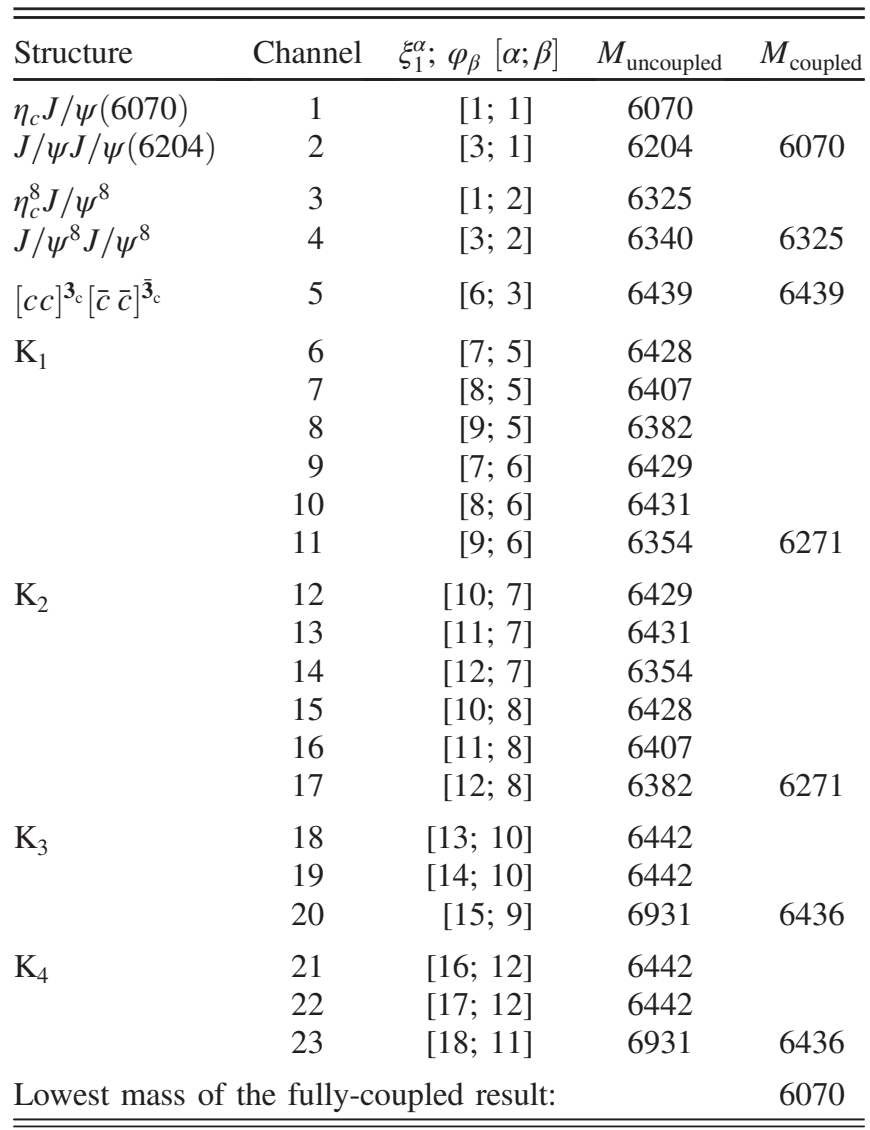

Fig. 3. While most of the complex eigenenergies decline with increasing angle $\theta$ indicating that they are scattering states of $\eta_{c} J / \psi$ and $J / \psi J / \psi$, we find three unchanged poles in the complex plane. Their masses and widths are (6274 MeV, $0.06 \mathrm{MeV})$, (6653 MeV, 1.20 MeV), and (6885 MeV, 4.10 MeV), respectively.

The resonance pole $(6885 \mathrm{MeV}, 4.10 \mathrm{MeV})$ can be identified as an $\eta_{c}(2 S) J / \psi(1 S)$ resonance. Its mass is quite close to that of the $\mathrm{X}(6900)$ structure seen by the LHCb collaboration. The other two resonances, $(6274 \mathrm{MeV}$, $0.06 \mathrm{MeV})$ and $(6653 \mathrm{MeV}, 1.20 \mathrm{MeV})$, can be identified with a $J / \psi(1 S) J / \psi(1 S)$ nature. They are located in the energy range where the broad structure is seen by the $\mathrm{LHCb}$ collaboration.

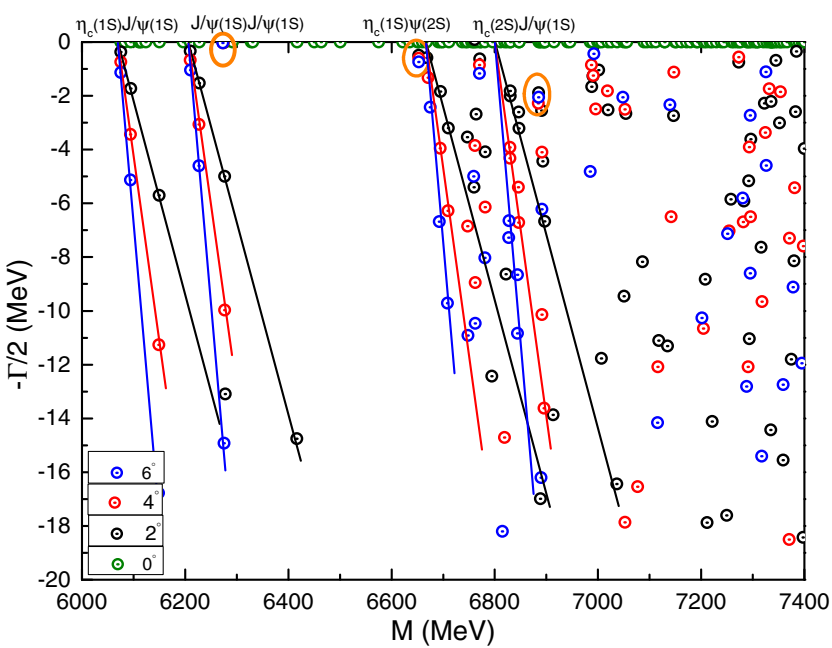

FIG. 3. Complex energy spectrum of the fully-charm tetraquark system with $J^{P(C)}=1^{+(-)}$from the complete coupled-channel calculation with CSM. The parameter $\theta$ varies from $0^{\circ}$ to $6^{\circ}$. Remember here that, according to the $\mathrm{ABC}$ theorem, (i) a bound state corresponds to a pole on the real axis whose energy is smaller than the meson-meson threshold and remains unchanged under the complex scaling transformation; (ii) the discretized continuum states are rotated downward by an angle of $2 \theta$ with respect the real axis; and (iii) a resonant state corresponds to a complex pole, that is independent of the angle $\theta$, circled in orange.

\section{The $J^{P(C)}=2^{+(+)}$channel}

We have two dimeson channels, $J / \psi J / \psi$ and $J / \psi^{8} J / \psi^{8}$, one diquark-antidiquark channel, $[c c]^{\mathbf{6}_{\mathrm{c}}}[\bar{c} \bar{c}]^{\overline{\boldsymbol{\sigma}}_{\mathrm{c}}}$, and six K-type ones, as listed in Table VII. Again, no bound state is found in this highest total-spin case by all kinds of computations, including the partially-coupled and fullycoupled ones. The lowest mass of the spectrum from the fully-coupled calculation is $6204 \mathrm{MeV}$, which is slightly above the theoretical threshold of di-J/ $/$.

Using the CSM, we obtain the complex energy spectrum from a complete coupled-channel calculation in Fig. 4. Therein, the nature of the scattering states, $J / \psi(1 S) J / \psi(1 S)$ and $J / \psi(1 S) \psi(2 S)$, are clearly shown by varying the angle $\theta$. One fixed pole at $(7007 \mathrm{MeV}$, $1.02 \mathrm{MeV}$ ) can be identified, and it can be regarded as a $J / \psi(1 S) \psi(2 S)$ resonance. Since the mass of this resonance is close to $6.9 \mathrm{GeV}$, the first radial excitation state of di$J / \psi$, i.e., $J / \psi(1 S) \psi(2 S)$, in $J^{P(C)}=2^{+(+)}$state is also a possible candidate for the $\mathrm{X}(6900)$.

\section{B. Fully-bottom system $\boldsymbol{b} b \bar{b} \bar{b}$}

We do not find bound states for all $S$-wave cases: $J^{P(C)}=0^{+(+)}, 1^{+(-)}$, and $2^{+(+)}$, of the fully-bottom system $b b \bar{b} \bar{b}$. However, performing a complete coupled-channel calculation within the CSM formalism, several narrow resonances are found and they are summarized in Table VIII. The results are discussed in the following. 
TABLE VII. Calculation of the $J^{P(C)}=2^{+(+)}$state of the fullycharm system in the real case $\left(\theta=0^{\circ}\right)$. The first and second columns show all possible structures and channels. They are obtained by the necessary bases in spin and color degrees of freedom summarized in the third column. The value in the parenthesis after the first meson-meson structure gives the theoretical result of the noninteracting meson-meson threshold. The lowest energies of all channels without considering any channel-channel coupling are listed in the fourth column. The last column shows the lowest energies in a calculation taking into account the couplings among the channels belonging to each structure (configuration). The last row gives the lowest energy from the complete coupled-channel calculation. All results are in units of $\mathrm{MeV}$.

\begin{tabular}{lcccc}
\hline \hline Structure & Channel & $\xi_{2} ; \varphi_{\beta}[\beta]$ & $M_{\text {uncoupled }}$ & $M_{\text {coupled }}$ \\
\hline$J / \psi J / \psi(6204)$ & 1 & 1 & 6204 & 6204 \\
$J / \psi^{8} J / \psi^{8}$ & 2 & 2 & 6388 & 6388 \\
{$[c c]^{3_{\mathrm{c}}}[\bar{c} \bar{c}]^{\overline{3}_{\mathrm{c}}}$} & 3 & 3 & 6472 & 6472 \\
$\mathrm{~K}_{1}$ & 4 & 5 & 6378 & \\
& 5 & 6 & 6374 & 6358 \\
$\mathrm{~K}_{2}$ & 6 & 7 & 6374 & \\
& 7 & 8 & 6378 & 6358 \\
$\mathrm{~K}_{3}$ & 8 & 10 & 6473 & 6473 \\
$\mathrm{~K}_{4}$ & 9 & 12 & 6473 & 6473 \\
Lowest mass of the fully-coupled result: & & 6204 \\
\hline \hline
\end{tabular}

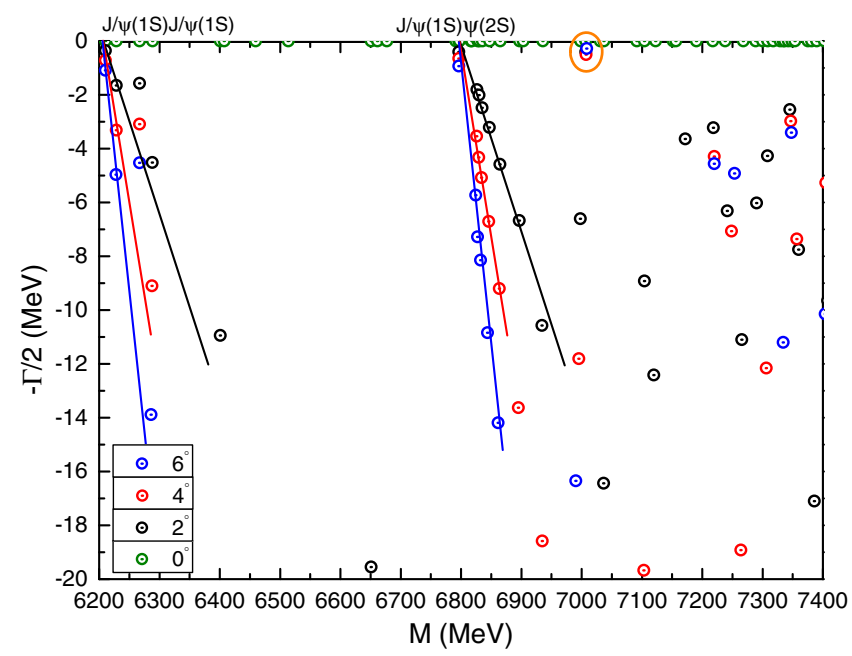

FIG. 4. Complex energy spectrum of the fully-charm tetraquark system with $J^{P(C)}=2^{+(+)}$from the complete coupled-channel calculation with CSM. The parameter $\theta$ varies from $0^{\circ}$ to $6^{\circ}$. Remember here that, according to the $\mathrm{ABC}$ theorem, (i) a bound state corresponds to a pole on the real axis whose energy is smaller than the meson-meson threshold and remains unchanged under the complex scaling transformation; (ii) the discretized continuum states are rotated downward by an angle of $2 \theta$ with respect the real axis; and (iii) a resonant state corresponds to a complex pole, that is independent of the angle $\theta$, circled in orange.
TABLE VIII. Predicted resonances of the fully-bottom tetraquark system from the complete coupled-channel calculation with CSM. Their masses and widths are summarized in the third and fourth column, respectively, in units of $\mathrm{MeV}$.

\begin{tabular}{cccc}
\hline \hline$J^{P(C)}$ & Resonance & Mass & Width \\
\hline $0^{+(+)}$ & $\eta_{b}(1 S) \eta_{b}(1 S)$ & 18882 & 1.42 \\
& $\Upsilon(1 S) \Upsilon(1 S)$ & 19019 & 1.28 \\
& $\eta_{b}(1 S) \eta_{b}(2 S)$ & 19394 & 0.16 \\
& $\Upsilon(1 S) \Upsilon(2 S)$ & 19454 & 0.46 \\
$1^{+(-)}$ & $\Upsilon(1 S) \Upsilon(1 S)$ & 19198 & 0.22 \\
& $\eta_{b}(1 S) \Upsilon(2 S)$ & 19402 & 0.22 \\
$2^{+(+)}$ & $\Upsilon(1 S) \Upsilon(2 S)$ & 19473 & 0.42 \\
& $\Upsilon(1 S) \Upsilon(2 S)$ & 19633 & 0.40 \\
\hline \hline
\end{tabular}

\section{The $J^{P(C)}=0^{+(+)}$channel}

Table IX lists the four dimeson channels, including the color-singlet and hidden-color configurations of $\eta_{b} \eta_{b}$ and

TABLE IX. Calculation of the $J^{P(C)}=0^{+(+)}$state of the fullybottom system in the real case $\left(\theta=0^{\circ}\right)$. The first and second columns show all possible structures and channels. They are obtained by the necessary bases in spin and color degrees of freedom summarized in the third column. Note also that, in the first column, when corresponding, the theoretical value of the noninteracting meson-meson threshold appears in parentheses. The lowest energies of all channels without considering any channel-channel coupling are listed in the fourth column. The last column shows the lowest energies in a calculation taking into account the couplings among the channels belonging to each structure (configuration). The last row gives the lowest energy from the complete coupled-channel calculation. All results are in units of MeV.

\begin{tabular}{|c|c|c|c|c|}
\hline Structure & Channel & $\xi_{0}^{\alpha} ; \varphi_{\beta}[\alpha ; \beta]$ & $M_{\text {uncoupled }}$ & $M_{\text {coupled }}$ \\
\hline$\eta_{b} \eta_{b}(18802)$ & 1 & {$[1 ; 1]$} & 18802 & \\
\hline$\Upsilon \Upsilon(18926)$ & 2 & {$[2 ; 1]$} & 18926 & 18802 \\
\hline$\eta_{b}^{8} \eta_{b}^{8}$ & 3 & {$[1 ; 2]$} & 19243 & \\
\hline$\Upsilon^{8} \Upsilon^{8}$ & 4 & {$[2 ; 2]$} & 19237 & 19144 \\
\hline$[b b]^{\mathbf{6}_{\mathrm{c}}}[\bar{b} \bar{b}]^{\overline{\mathbf{6}}_{\mathrm{c}}}$ & 5 & {$[3 ; 4]$} & 19173 & \\
\hline$[b b]^{\mathbf{3}_{\mathrm{c}}}[\bar{b} \bar{b}]^{\mathbf{3}_{\mathrm{c}}}$ & 6 & {$[4 ; 3]$} & 19196 & 19148 \\
\hline \multirow[t]{4}{*}{$\mathrm{K}_{1}$} & 7 & {$[5 ; 5]$} & 19237 & \\
\hline & 8 & {$[5 ; 6]$} & 19058 & \\
\hline & 9 & {$[6 ; 5]$} & 19241 & \\
\hline & 10 & {$[6 ; 6]$} & 18992 & 18977 \\
\hline \multirow[t]{4}{*}{$\mathrm{K}_{2}$} & 11 & {$[7 ; 7]$} & 19058 & \\
\hline & 12 & {$[7 ; 8]$} & 19237 & \\
\hline & 13 & {$[8 ; 7]$} & 18992 & \\
\hline & 14 & {$[8 ; 8]$} & 19241 & 18977 \\
\hline \multirow[t]{2}{*}{$\mathrm{K}_{3}$} & 15 & {$[9 ; 10]$} & 19197 & \\
\hline & 16 & {$[10 ; 9]$} & 19167 & 19143 \\
\hline \multirow[t]{2}{*}{$\mathrm{K}_{4}$} & 17 & {$[11 ; 12]$} & 19197 & \\
\hline & 18 & {$[12 ; 11]$} & 19167 & 19143 \\
\hline \multicolumn{4}{|c|}{ Lowest mass of the fully-coupled result: } & 18802 \\
\hline
\end{tabular}


$\Upsilon \Upsilon$, the two diquark-antidiquark channels, $[b b]^{\mathbf{3}_{\mathrm{c}}}[\bar{b} \bar{b}]^{\overline{\mathbf{3}}_{\mathrm{c}}}$ and $[b b]^{\boldsymbol{6}_{\mathrm{c}}}[\bar{b} \bar{b}]^{\mathbf{\boldsymbol { b }}_{\mathrm{c}}}$, and twelve K-type allowed structures. For the color-singlet channels of di- $\eta_{b}$ and di- $\Upsilon$ configurations, the lowest masses are just their corresponding theoretical thresholds, $18802 \mathrm{MeV}$ and $18926 \mathrm{MeV}$, respectively. For other exotic structures, most of their masses are above $19.1 \mathrm{GeV}$, while two channels in the $\mathrm{K}_{1}$ and $\mathrm{K}_{2}$ configurations possess masses around $19 \mathrm{GeV}$, close to the threshold of the $\Upsilon \Upsilon$ channel. When a partially-coupled calculation is performed for each structure, masses are ranging from 18.8 to $19.1 \mathrm{GeV}$. The lowest mass of the spectrum from the fully-coupled calculation is $18802 \mathrm{MeV}$, which is slightly above the threshold of di- $\eta_{b}$. Therefore, no bound state exists for $J^{P(C)}=0^{+(+)}$.

We now look for possible resonances by performing a complete coupled-channel calculation within CSM. The complex energy spectrum is presented in Fig. 5, with energy ranging from 18.8 to $19.5 \mathrm{GeV}$. There are four scattering states, $\eta_{b}(1 S) \eta_{b}(1 S), \quad \Upsilon(1 S) \Upsilon(1 S)$, $\eta_{b}(1 S) \eta_{b}(2 S)$, and $\Upsilon(1 S) \Upsilon(2 S)$; with varying angle $\theta$, the complex eigenenergies are generally aligned along the cut lines. In addition to the scattering states, four resonance poles are found whose structure and mass can be identified with $\eta_{b}(1 S) \eta_{b}(1 S)(18882), \quad \Upsilon(1 S) \Upsilon(1 S)(19019)$, $\eta_{b}(1 S) \eta_{b}(2 S)(19394)$ and $\Upsilon(1 S) \Upsilon(2 S)(19454)$. Their widths are small, they are $1.42 \mathrm{MeV}, 1.28 \mathrm{MeV}$, $0.16 \mathrm{MeV}$, and $0.46 \mathrm{MeV}$, respectively.

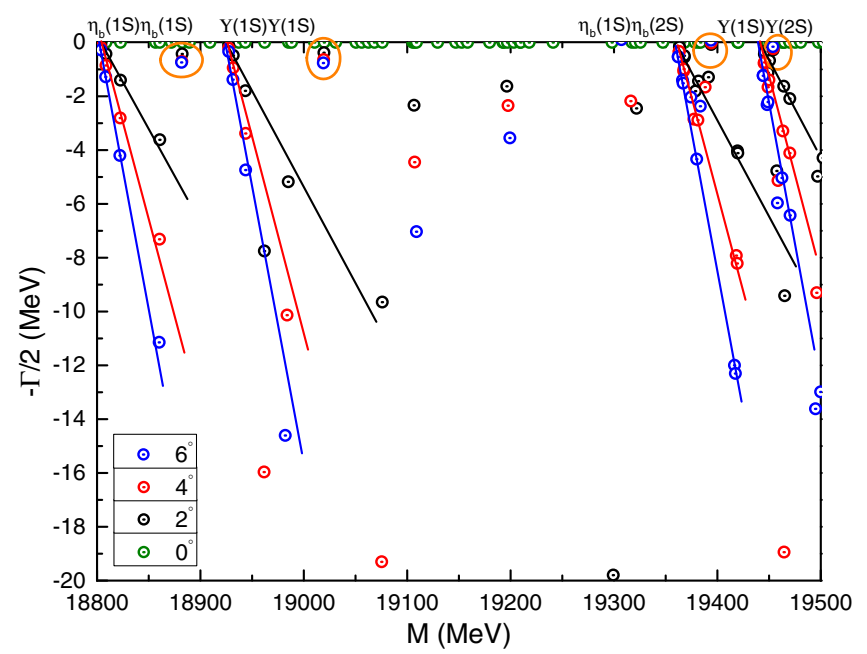

FIG. 5. Complex energy spectrum of the fully-bottom tetraquark system with $J^{P(C)}=0^{+(+)}$from the complete coupledchannel calculation with CSM. The parameter $\theta$ varies from $0^{\circ}$ to $6^{\circ}$. Remember here that, according to the ABC theorem, (i) a bound state corresponds to a pole on the real axis whose energy is smaller than the meson-meson threshold and remains unchanged under the complex scaling transformation; (ii) the discretized continuum states are rotated downward by an angle of $2 \theta$ with respect the real axis; and (iii) a resonant state corresponds to a complex pole, that is independent of the angle $\theta$, circled in orange.
Although the fully-bottom tetraquark state has not yet been reported experimentally, our theoretical findings could be valuable for future experimental studies. In fact, the $\Upsilon(1 S) \Upsilon(1 S)(19019)$ resonance is also supported in other theoretical works as, for instance, Ref. [52].

\section{The $J^{P(C)}=1^{+(-)}$channel}

There are 23 channels listed in Table $\mathrm{X}$, including four dimeson channels with $\eta_{b} \Upsilon$ and $\Upsilon \Upsilon$ dimeson configurations in the singlet- and hidden-color states, one diquark-antidiquark channel, $[b b]^{\mathbf{6}_{\mathrm{c}}}[\bar{b} \bar{b}]^{\overline{\boldsymbol{\sigma}}_{\mathrm{c}}}$, and $18 \mathrm{~K}$-type ones. First of all, the lowest channel $\eta_{b} \Upsilon$ is unbound, with a mass of $18864 \mathrm{MeV}$. The other color-singlet dimeson

TABLE X. Calculation of the $J^{P(C)}=1^{+(-)}$state of the fullybottom system in the real case $\left(\theta=0^{\circ}\right)$. The first and second columns show all possible structures and channels. They are obtained by the necessary bases in spin and color degrees of freedom summarized in the third column. Note also that, in the first column, when corresponding, the theoretical value of the noninteracting meson-meson threshold appears in parentheses. The lowest energies of all channels without considering any channel-channel coupling are listed in the fourth column. The last column shows the lowest energies in a calculation taking into account the couplings among the channels belonging to each structure (configuration). The last row gives the lowest energy from the complete coupled-channel calculation. All results are in units of MeV.

\begin{tabular}{lcccc}
\hline \hline Structure & Channel & $\xi_{1}^{\alpha} ; \varphi_{\beta}[\alpha ; \beta]$ & $M_{\text {uncoupled }}$ & $M_{\text {coupled }}$ \\
\hline$\eta_{b} \Upsilon(18864)$ & 1 & {$[1 ; 1]$} & 18864 & \\
$\Upsilon \Upsilon(18926)$ & 2 & {$[3 ; 1]$} & 18926 & 18864 \\
$\eta_{b}^{8} \Upsilon^{8}$ & 3 & {$[1 ; 2]$} & 19126 & \\
$\Upsilon^{8} \Upsilon^{8}$ & 4 & {$[3 ; 2]$} & 19227 & 19126 \\
{$[b b]^{3_{c}}[\bar{b} \bar{b}]^{\mathbf{3}_{c}}$} & 5 & {$[6 ; 3]$} & 19205 & 19205 \\
$\mathrm{~K}_{1}$ & 6 & {$[7 ; 5]$} & 19197 & \\
& 7 & {$[8 ; 5]$} & 19191 & \\
& 8 & {$[9 ; 5]$} & 19170 & \\
& 9 & {$[7 ; 6]$} & 19186 & \\
& 10 & {$[8 ; 6]$} & 19197 & \\
$\mathrm{~K}_{2}$ & 11 & {$[9 ; 6]$} & 19139 & 19053 \\
& 12 & {$[10 ; 7]$} & 19186 & \\
& 13 & {$[11 ; 7]$} & 19197 & \\
& 14 & {$[12 ; 7]$} & 19139 & \\
& 15 & {$[10 ; 8]$} & 19197 & \\
& 16 & {$[11 ; 8]$} & 19191 & \\
$\mathrm{~K}_{3}$ & 17 & {$[12 ; 8]$} & 19170 & 19053 \\
& 18 & {$[13 ; 10]$} & 19212 & \\
$\mathrm{~K}_{4}$ & 19 & {$[14 ; 10]$} & 19211 & \\
& 20 & {$[15 ; 9]$} & 19603 & 19206 \\
Lowest mass of the fully-coupled result: & & 18864 \\
\hline \hline
\end{tabular}


channel, $\Upsilon$, is also unbound with the lowest mass $18926 \mathrm{MeV}$. The masses of the remaining configurations populate densely a small energy range, 19.1-19.2 GeV; except for two channels from $\mathrm{K}_{3}$ and $\mathrm{K}_{4}$ structures whose masses are located at $\sim 19.6 \mathrm{GeV}$. The lowest mass of the spectrum from the fully-coupled calculation is $18864 \mathrm{MeV}$, which is slightly above the threshold of $\eta_{b} \Upsilon$. Therefore, no bound state exists for $J^{P(C)}=1^{+(-)}$.

Again, we look for possible resonances by performing a complete coupled-channel calculation with CSM. The complex energy spectrum is shown in Fig. 6. Apart from four continuum states, $\eta_{b}(1 S) \Upsilon(1 S), \quad \Upsilon(1 S) \Upsilon(1 S)$, $\eta_{b}(1 S) \Upsilon(2 S)$, and $\eta_{b}(2 S) \Upsilon(1 S)$, we find two resonance poles in the complex energy plane. Attending to their components and masses, we can identify these two resonance poles as $\Upsilon(1 S) \Upsilon(1 S)(19198)$ and $\eta_{b}(1 S) \Upsilon(2 S)(19402)$. Both have extremely small two-meson decay widths: $0.22 \mathrm{MeV}$.

\section{The $J^{P(C)}=2^{+(+)}$channel}

We have 9 channels in this case, as shown in Table XI, including two dimeson channels, $\Upsilon \Upsilon$ configurations in the singlet- and hidden-color states, one diquark-antidiquark channel $[b b]^{\mathbf{6}_{\mathrm{c}}}[\bar{b} \bar{b}]^{\overline{6}_{\mathrm{c}}}$, and six K-type ones. The calculated lowest mass for $\Upsilon \Upsilon$ is $18926 \mathrm{MeV}$, which is equal to its theoretical threshold. The masses of the other channels are all above $19.1 \mathrm{GeV}$, and there is an approximate degeneracy

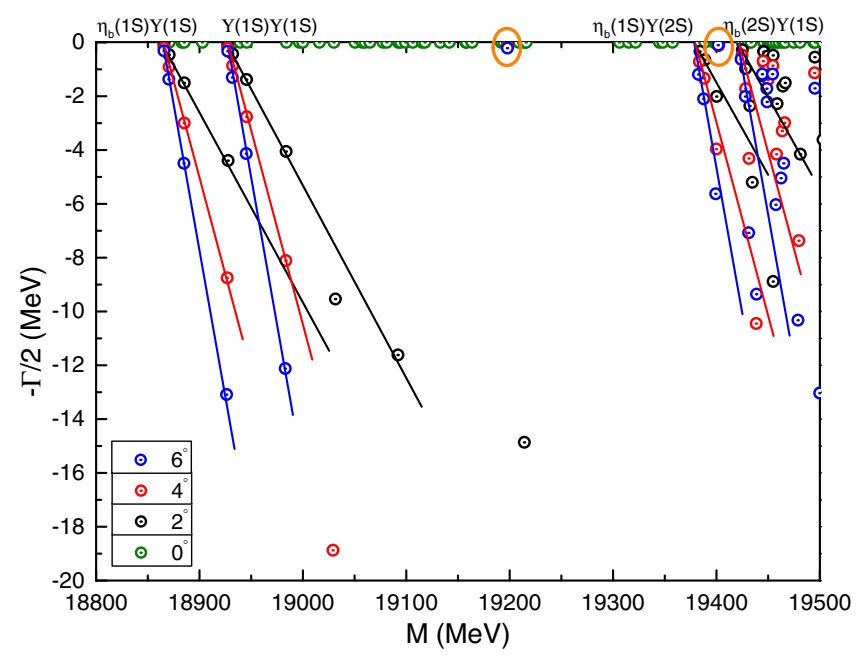

FIG. 6. Complex energy spectrum of the fully-bottom tetraquark system with $J^{P(C)}=1^{+(-)}$from the complete coupledchannel calculation with CSM. The parameter $\theta$ varies from $0^{\circ}$ to $6^{\circ}$. Remember here that, according to the ABC theorem, (i) a bound state corresponds to a pole on the real axis whose energy is smaller than the meson-meson threshold and remains unchanged under the complex scaling transformation; (ii) the discretized continuum states are rotated downward by an angle of $2 \theta$ with respect the real axis; and (iii) a resonant state corresponds to a complex pole, that is independent of the angle $\theta$, circled in orange.

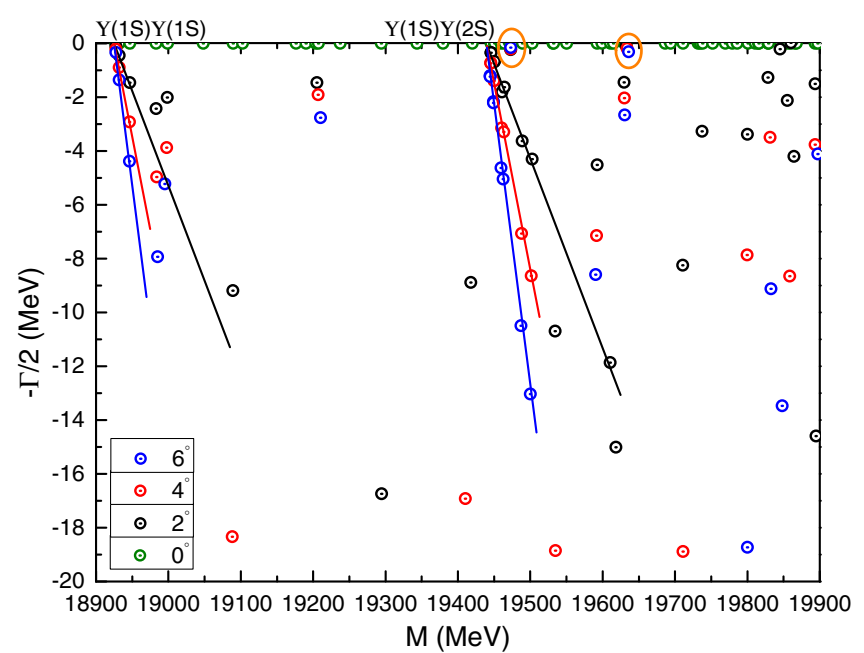

FIG. 7. Complex energy spectrum of the fully-bottom tetraquark system with $J^{P(C)}=2^{+(+)}$from the complete coupledchannel calculation with CSM. The parameter $\theta$ varies from $0^{\circ}$ to $6^{\circ}$. Remember here that, according to the $\mathrm{ABC}$ theorem, (i) a bound state corresponds to a pole on the real axis whose energy is smaller than the meson-meson threshold and remains unchanged under the complex scaling transformation; (ii) the discretized continuum states are rotated downward by an angle of $2 \theta$ with respect the real axis; and (iii) a resonant state corresponds to a complex pole, that is independent of the angle $\theta$, circled in orange.

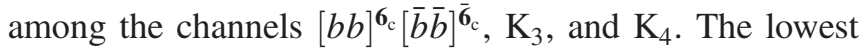
mass of the spectrum from the fully-coupled calculation is $18926 \mathrm{MeV}$, which is slightly above the threshold of $\Upsilon \Upsilon$. Therefore, no bound state exists for $J^{P(C)}=2^{+(+)}$.

Fig. 7 shows the complex energy spectrum for $J^{P(C)}=2^{+(+)}$, obtained by using CSM. We find two resonance poles above the $\Upsilon(1 S) \Upsilon(2 S)$ threshold. They can be identified as $\Upsilon(1 S) \Upsilon(2 S)(19473)$ and $\Upsilon(1 S) \Upsilon(2 S)$ (19633), with their widths $0.42 \mathrm{MeV}$ and $0.40 \mathrm{MeV}$, respectively.

\section{Charm-bottom system $\bar{c} c \bar{c} b$}

For the charm-bottom system $\bar{c} c \bar{c} b$, narrow resonances are found in $J^{P}=0^{+}, 1^{+}$, and $2^{+}$states, respectively. Table XII summarizes the results and the details are discussed in the following.

\section{The $J^{P}=0^{+}$channel}

Table XIII lists the lowest masses of each four configurations of $\bar{c} c \bar{c} b$ tetraquark in $0^{+(+)}$states. In the real-range calculation, $\theta=0^{\circ}$, the two dimeson color-singlet channels $\eta_{c} B_{c}$ and $J / \psi B_{c}^{*}$ are just scattering states, and their hiddencolor cases present masses about $9.6 \mathrm{GeV}$. The two diquark-antidiquark structures are almost degenerate at $9.67 \mathrm{GeV}$. As for the other K-type configurations, which are all excited channels, the calculated single-channel masses are generally in a mass region of $9.45-9.69 \mathrm{GeV}$. 
TABLE XI. Calculation of the $J^{P(C)}=2^{+(+)}$state of the fullybottom system in the real case $\left(\theta=0^{\circ}\right)$. The first and second columns show all possible structures and channels. They are obtained by the necessary bases in spin and color degrees of freedom summarized in the third column. The value in the parenthesis after the first meson-meson structure gives the theoretical result of the noninteracting meson-meson threshold. The lowest energies of all channels without considering any channel-channel coupling are listed in the fourth column. The last column shows the lowest energies in a calculation taking into account the couplings among the channels belonging to each structure (configuration). The last row gives the lowest energy from the complete coupled-channel calculation. All results are in units of MeV.

\begin{tabular}{|c|c|c|c|c|}
\hline Structure & Channel & $\xi_{2} ; \varphi_{\beta}[\beta]$ & $M_{\text {uncoupled }}$ & $M_{\text {coupled }}$ \\
\hline$\Upsilon \Upsilon(18926)$ & 1 & 1 & 18926 & 18926 \\
\hline$\Upsilon^{8} \Upsilon^{8}$ & 2 & 2 & 19197 & 19197 \\
\hline$[b b]^{\mathbf{3}_{\mathrm{c}}}[\bar{b} \bar{b}]^{\mathbf{3}_{\mathrm{c}}}$ & 3 & 3 & 19223 & 19223 \\
\hline \multirow[t]{2}{*}{$\mathrm{K}_{1}$} & 4 & 5 & 19147 & \\
\hline & 5 & 6 & 19101 & 19093 \\
\hline \multirow[t]{2}{*}{$\mathrm{K}_{2}$} & 6 & 7 & 19101 & \\
\hline & 7 & 8 & 19147 & 19093 \\
\hline $\mathrm{K}_{3}$ & 8 & 10 & 19225 & 19225 \\
\hline $\mathrm{K}_{4}$ & 9 & 12 & 19225 & 19225 \\
\hline \multicolumn{4}{|c|}{ Lowest mass of the fully-coupled result: } & 18926 \\
\hline
\end{tabular}

Then, in the coupled-channel investigations on each configuration, bound states are still not found, the lowest mass is just the theoretical threshold value of $\eta_{c} B_{c}$ at $9243 \mathrm{MeV}$, and the other excited configurations are generally at $9.5 \mathrm{GeV}$. This fact remains in a complete coupled-channels calculation, whose lowest mass at $9243 \mathrm{MeV}$ is shown in the last row of Table XIII.

In a further step, when a complex-scaling study is considered in a fully-coupled calculation, one narrow resonance state is found. Figure 8 presents the distributions of the complex energies of $\bar{c} c \bar{c} b$ tetraquarks with rotated angle $\theta$ varied from $0^{\circ}$ to $6^{\circ}$. In a mass region from $9.2 \mathrm{GeV}$

TABLE XII. Predicted resonances of the $\bar{c} c \bar{c} b$ tetraquark system from the complete coupled-channel calculation with CSM. Their masses and widths are summarized in the third and fourth column, respectively, in units of $\mathrm{MeV}$.

\begin{tabular}{lccc}
\hline \hline$J^{P}$ & Resonance & Mass & Width \\
\hline $0^{+}$ & $J / \psi(1 S) B_{c}^{*}(1 S)$ & 9813 & 4.02 \\
$1^{+}$ & $J / \psi(1 S) B_{c}^{*}(1 S)$ & 9823 & 2.41 \\
& $\eta_{c}(1 S) B_{c}^{*}(2 S)$ & 9898 & 1.33 \\
& $\eta_{c}(1 S) B_{c}^{*}(2 S)$ & 9928 & 3.80 \\
& $\eta_{c}(1 S) B_{c}^{*}(2 S)$ & 9974 & 8.21 \\
& $\eta_{c}(2 S) B_{c}^{*}(1 S)$ & 10284 & 3.00 \\
$2^{+}$ & $\psi(2 S) B_{c}^{*}(1 S)$ & 10563 & 6.61 \\
\hline \hline
\end{tabular}

TABLE XIII. Calculation of the $J^{P}=0^{+}$state of the $\bar{c} c \bar{c} b$ system in the real case $\left(\theta=0^{\circ}\right)$. The first and second columns show all possible structures and channels. They are obtained by the necessary bases in spin and color degrees of freedom summarized in the third column. Note also that, in the first column, when corresponding, the theoretical value of the noninteracting meson-meson threshold appears in parentheses. The lowest energies of all channels without considering any channelchannel coupling are listed in the fourth column. The last column shows the lowest energies in a calculation taking into account the couplings among the channels belonging to each structure (configuration). The last row gives the lowest energy from the complete coupled-channel calculation. All results are in units of $\mathrm{MeV}$.

\begin{tabular}{|c|c|c|c|c|}
\hline Structure & Channel & $\xi_{0}^{\alpha} ; \varphi_{\beta}[\alpha ; \beta]$ & $M_{\text {uncoupled }}$ & $M_{\text {coupled }}$ \\
\hline$\eta_{c} B_{c}(9243)$ & 1 & {$[1 ; 1]$} & 9243 & \\
\hline$J / \psi B_{c}^{*}(9451)$ & 2 & {$[2 ; 1]$} & 9451 & 9243 \\
\hline$\eta_{c}^{8} B_{c}^{8}$ & 3 & {$[1 ; 2]$} & 9683 & \\
\hline$J / \psi^{8} B_{c}^{* 8}$ & 4 & {$[2 ; 2]$} & 9625 & 9559 \\
\hline 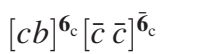 & 5 & {$[3 ; 4]$} & 9672 & \\
\hline$[c b]^{\mathbf{3}_{\mathrm{c}}}[\bar{c} \bar{c}]^{\mathbf{3}_{\mathrm{c}}}$ & 6 & {$[4 ; 3]$} & 9673 & 9620 \\
\hline \multirow[t]{4}{*}{$\mathrm{K}_{1}$} & 7 & {$[5 ; 5]$} & 9617 & \\
\hline & 8 & {$[5 ; 6]$} & 9696 & \\
\hline & 9 & {$[6 ; 5]$} & 9690 & \\
\hline & 10 & {$[6 ; 6]$} & 9558 & 9551 \\
\hline \multirow[t]{4}{*}{$\mathrm{K}_{2}$} & 11 & {$[7 ; 7]$} & 9602 & \\
\hline & 12 & {$[7 ; 8]$} & 9624 & \\
\hline & 13 & {$[8 ; 7]$} & 9447 & \\
\hline & 14 & {$[8 ; 8]$} & 9699 & 9310 \\
\hline \multirow[t]{4}{*}{$\mathrm{K}_{3}$} & 15 & {$[9 ; 9]$} & 10105 & \\
\hline & 16 & {$[9 ; 10]$} & 9673 & \\
\hline & 17 & {$[10 ; 9]$} & 9666 & \\
\hline & 18 & {$[10 ; 10]$} & 10172 & 9599 \\
\hline \multirow[t]{2}{*}{$\mathrm{K}_{4}$} & 19 & {$[11 ; 12]$} & 9672 & \\
\hline & 20 & {$[12 ; 11]$} & 9689 & 9621 \\
\hline \multicolumn{4}{|c|}{ Lowest mass of the fully-coupled result: } & 9243 \\
\hline
\end{tabular}

to $10.2 \mathrm{GeV}$, the scattering nature of $\eta_{c} B_{c}$ and $J / \psi B_{c}^{*}$ both in the ground and first radial excitation states are well established. One can also see in Fig. 8 a stable resonance pole, whose mass and width are $9813 \mathrm{MeV}$ and $4.02 \mathrm{MeV}$, respectively. This resonance is below the threshold lines of $\eta_{c}(1 S) B_{c}(2 S)$, and the dominant channel, therefore, can be identified as the $J / \psi(1 S) B_{c}^{*}(1 S)$ state.

\section{E. The $J^{P}=1^{+}$channel}

There are in all 30 channels listed in Table XIV to be considered for the $\bar{c} c \bar{c} b$ tetraquark in $1^{+}$state. In particular, six channels devote to the meson-meson structures, three channels belong to the diquark-antidiquark ones, and 21 channels correspond to the K-type configurations. When they are considered as isolated channels or when a coupledchannels calculation in each configurations is performed, 


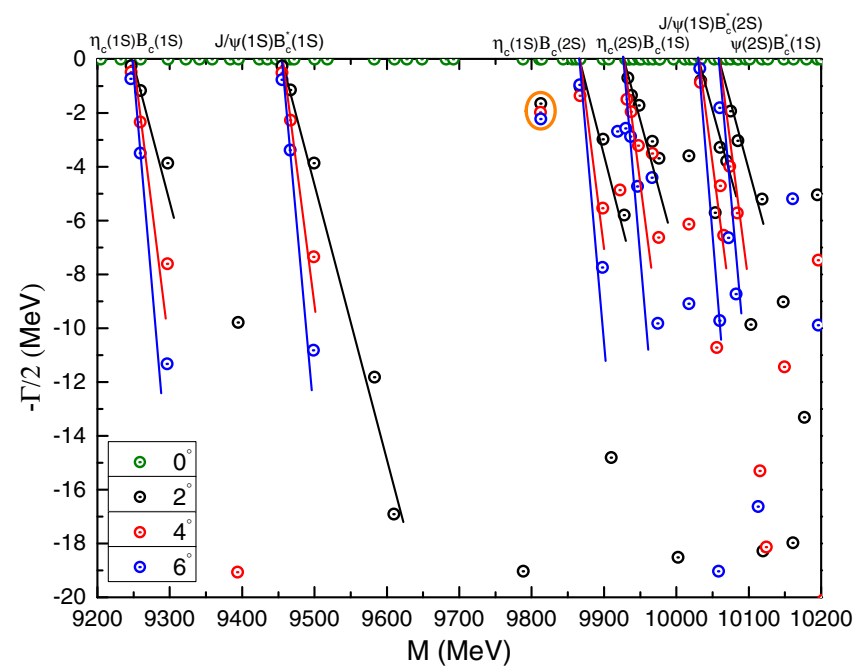

FIG. 8. Complex energy spectrum of the $\bar{c} c \bar{c} b$ system with $J^{P}=0^{+}$from the complete coupled-channel calculation with CSM. The parameter $\theta$ varies from $0^{\circ}$ to $6^{\circ}$. Remember here that, according to the $\mathrm{ABC}$ theorem, (i) a bound state corresponds to a pole on the real axis whose energy is smaller than the mesonmeson threshold and remains unchanged under the complex scaling transformation; (ii) the discretized continuum states are rotated downward by an angle of $2 \theta$ with respect the real axis; and (iii) a resonant state corresponds to a complex pole, that is independent of the angle $\theta$, circled in orange.

no bound state is found. The lowest mass, $9317 \mathrm{MeV}$, is the theoretical threshold value of $\eta_{c} B_{c}^{*}$; the other two dimeson channels, $J / \psi B_{c}$ and $J / \psi B_{c}^{*}$, are at $9377 \mathrm{MeV}$ and $9451 \mathrm{MeV}$, respectively; the exotic configurations, i.e., hidden-color, diquark-antidiquark and K-types structures, are generally located in a energy range from $9.62 \mathrm{GeV}$ to $9.68 \mathrm{GeV}$; and the lowest mass in each of the configuration's coupled-channels calculation is around $9.60 \mathrm{GeV}$.

However, several resonances are obtained when the complex scaling method is employed in a complete coupled-channels calculation. Figure 9 presents our results; in particular, the top panel shows the distributions of $\eta_{c} B_{c}^{*}$, $J / \psi B_{c}$, and $J / \psi B_{c}^{*}$ continuum states, their ground and first radial excitation states basically align along the corresponding threshold lines. Nevertheless, two stable resonance poles are circled therein and they can be identified as $J / \psi(1 S) B_{c}^{*}(1 S)(9823)$ and $\eta_{c}(2 S) B_{c}^{*}(1 S)(10284)$ states, with resonance's widths $2.41 \mathrm{MeV}$ and $3.00 \mathrm{MeV}$, respectively.

Since the first radial excitation of $\eta_{c} B_{c}^{*}, J / \psi B_{c}$, and $J / \psi B_{c}^{*}$ states are densely distribute in the top panel of Fig. 9 , an enlarged zoom, with energy range from $9.85 \mathrm{GeV}$ to $10.05 \mathrm{GeV}$, is presented as bottom panel. Therein, the first radial excitation states of $\eta_{c}(1 S) B_{c}^{*}(2 S), J / \psi(1 S) B_{c}(2 S)$, $\psi(2 S) B_{c}(1 S)$, and $\eta_{c}(2 S) B_{c}^{*}(1 S)$ are clearly shown. Additionally, three more $\eta_{c}(1 S) B_{c}^{*}(2 S)$ resonances are found, i.e., $\quad \eta_{c}(1 S) B_{c}^{*}(2 S)(9898), \quad \eta_{c}(1 S) B_{c}^{*}(2 S)(9928)$
TABLE XIV. Calculation of the $J^{P}=1^{+}$state of the $\bar{c} c \bar{c} b$ system in the real case $\left(\theta=0^{\circ}\right)$. The first and second columns show all possible structures and channels. They are obtained by the necessary bases in spin and color degrees of freedom summarized in the third column. Note also that, in the first column, when corresponding, the theoretical value of the noninteracting meson-meson threshold appears in parentheses. The lowest energies of all channels without considering any channelchannel coupling are listed in the fourth column. The last column shows the lowest energies in a calculation taking into account the couplings among the channels belonging to each structure (configuration). The last row gives the lowest energy from the complete coupled-channel calculation. All results are in units of $\mathrm{MeV}$.

\begin{tabular}{|c|c|c|c|c|}
\hline Structure & Channel & $\xi_{1}^{\alpha} ; \varphi_{\beta}[\alpha ; \beta]$ & $M_{\text {uncoupled }}$ & $M_{\text {coupled }}$ \\
\hline$\eta_{c} B_{c}^{*}(9317)$ & 1 & {$[1 ; 1]$} & 9317 & \\
\hline$J / \psi B_{c}(9377)$ & 2 & {$[2 ; 1]$} & 9377 & \\
\hline$J / \psi B_{c}^{*}(9451)$ & 3 & {$[3 ; 1]$} & 9451 & 9317 \\
\hline$\eta_{c}^{8} B_{c}^{* 8}$ & 4 & {$[1 ; 2]$} & 9659 & \\
\hline$J / \psi^{8} B_{c}^{8}$ & 5 & {$[2 ; 2]$} & 9651 & \\
\hline$J / \psi^{8} B_{c}^{* 8}$ & 6 & {$[3 ; 2]$} & 9622 & 9587 \\
\hline$[c b]^{\mathbf{6}_{\mathrm{c}}}[\bar{c} \bar{c}]^{\mathbf{6}_{\mathrm{c}}}$ & 7 & {$[4 ; 4]$} & 9665 & \\
\hline$[c b]^{\mathbf{3}_{\mathrm{c}}}[\bar{c} \bar{c}]^{\overline{\mathbf{3}}_{\mathrm{c}}}$ & 8 & {$[5 ; 3]$} & 9675 & \\
\hline$[c b]^{\mathbf{3}_{\mathrm{c}}}[\bar{c} \bar{c}]^{\mathbf{3}_{\mathrm{c}}}$ & 9 & {$[6 ; 3]$} & 9687 & 9638 \\
\hline $\mathrm{K}_{1}$ & 10 & {$[7 ; 5]$} & 9671 & \\
\hline & 11 & {$[8 ; 5]$} & 9619 & \\
\hline & 12 & {$[9 ; 5]$} & 9668 & \\
\hline & 13 & {$[7 ; 6]$} & 9673 & \\
\hline & 14 & {$[8 ; 6]$} & 9676 & \\
\hline & 15 & {$[9 ; 6]$} & 9577 & 9568 \\
\hline $\mathrm{K}_{2}$ & 16 & {$[10 ; 7]$} & 9574 & \\
\hline & 17 & {$[11 ; 7]$} & 9599 & \\
\hline & 18 & {$[12 ; 7]$} & 9650 & \\
\hline & 19 & {$[10 ; 8]$} & 9636 & \\
\hline & 20 & {$[11 ; 8]$} & 9633 & \\
\hline & 21 & {$[12 ; 8]$} & 9674 & 9546 \\
\hline $\mathrm{K}_{3}$ & 22 & {$[13 ; 9]$} & 9681 & \\
\hline & 23 & {$[14 ; 9]$} & 9678 & \\
\hline & 24 & {$[15 ; 9]$} & 10165 & \\
\hline & 25 & {$[13 ; 10]$} & 9696 & \\
\hline & 26 & {$[14 ; 10]$} & 9696 & \\
\hline & 27 & {$[15 ; 10]$} & 9677 & 9619 \\
\hline $\mathrm{K}_{4}$ & 28 & {$[16 ; 12]$} & 9689 & \\
\hline & 29 & {$[17 ; 12]$} & 9679 & \\
\hline & 30 & {$[18 ; 11]$} & 9678 & 9640 \\
\hline \multicolumn{4}{|c|}{ Lowest mass of the fully-coupled result: } & 9317 \\
\hline
\end{tabular}

and $\eta_{c}(1 S) B_{c}^{*}(2 S)(9974)$, their resonance widths are $1.33 \mathrm{MeV}, 3.80 \mathrm{MeV}$, and $8.21 \mathrm{MeV}$, respectively.

\section{F. The $J^{P}=2^{+}$channel}

We have ten channels listed in Table XV. There is only one channel for the dimeson structure, $J / \psi B_{c}^{*}$, another one 

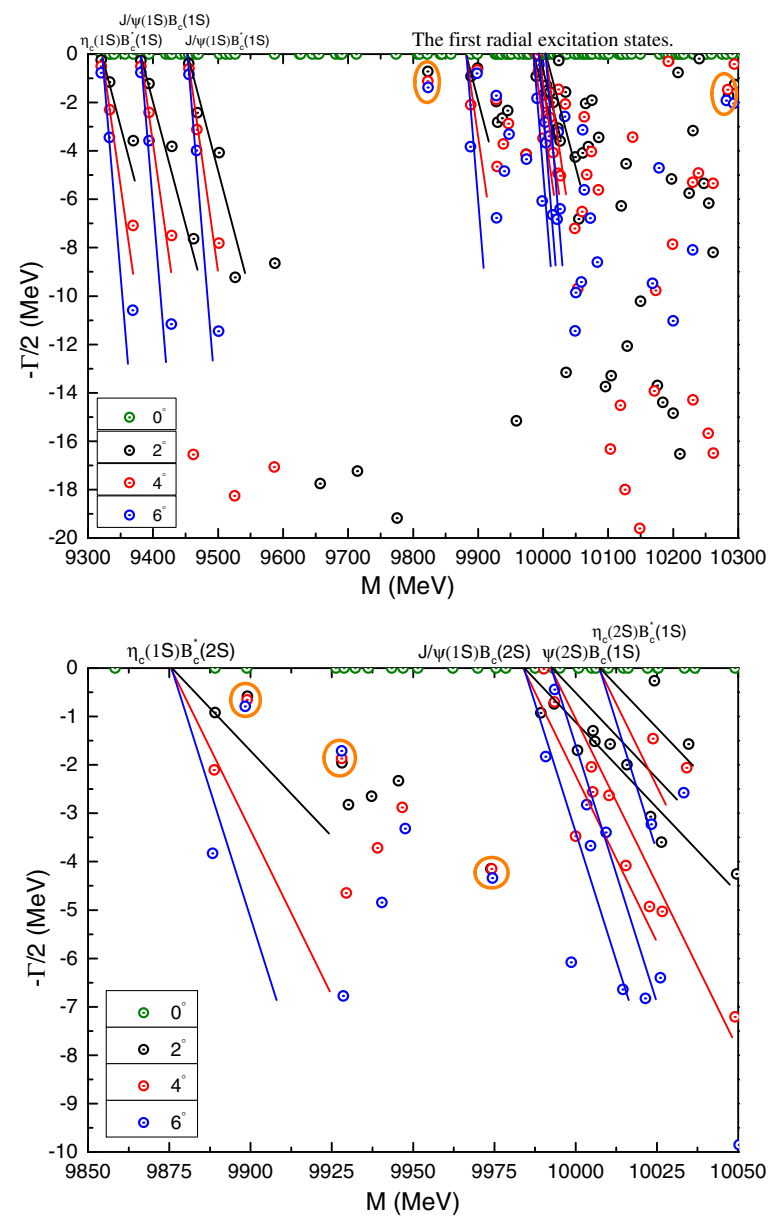

FIG. 9. Top panel: complex energy spectrum of the $\bar{c} c \bar{c} b$ system with $J^{P}=1^{+}$from the complete coupled-channel calculation with CSM. The parameter $\theta$ varies from $0^{\circ}$ to $6^{\circ}$. Bottom panel: enlarged case of the top panel, with real values of energy ranging from $9.85 \mathrm{GeV}$ to $10.05 \mathrm{GeV}$. Remember here that, according to the $\mathrm{ABC}$ theorem, (i) a bound state corresponds to a pole on the real axis whose energy is smaller than the mesonmeson threshold and remains unchanged under the complex scaling transformation; (ii) the discretized continuum states are rotated downward by an angle of $2 \theta$ with respect the real axis; and (iii) a resonant state corresponds to a complex pole, that is independent of the angle $\theta$, circled in orange.

for the diquark-antidiquark case, $[c b]^{3 c}[\bar{c} \bar{c}]^{\overline{3} c}$, and the rest belong to the K-type configurations. We obtain a lowest mass of $9451 \mathrm{MeV}$, which is just the theoretical threshold value of $J / \psi B_{c}^{*}$. Generally, the unconventional structures have masses which lie within the range 9.65-9.71 GeV. Moreover, no bound state is found when coupled-channel effects are taken into account in each tetraquark configuration separately.

Figure 10 shows the distribution of the complex energies obtained for the $\bar{c} c \bar{c} b$ tetraquark system. The nature as scattering states of the $J / \psi B_{c}^{*}$ system in ground and first radial excitation are well shown. However, from $9.45 \mathrm{GeV}$ to $10.65 \mathrm{GeV}$ energy region, a narrow resonance pole is
TABLE XV. Calculation of the $J^{P}=2^{+}$state of the $\bar{c} c \bar{c} b$ system in the real case $\left(\theta=0^{\circ}\right)$. The first and second columns show all possible structures and channels. They are obtained by the necessary bases in spin and color degrees of freedom summarized in the third column. Note also that, in the first column, when corresponding, the theoretical value of the noninteracting meson-meson threshold appears in parentheses. The lowest energies of all channels without considering any channelchannel coupling are listed in the fourth column. The last column shows the lowest energies in a calculation taking into account the couplings among the channels belonging to each structure (configuration). The last row gives the lowest energy from the complete coupled-channel calculation. All results are in units of $\mathrm{MeV}$.

\begin{tabular}{lcccc}
\hline \hline Structure & Channel & $\xi_{2} ; \varphi_{\beta}[\beta]$ & $M_{\text {uncoupled }}$ & $M_{\text {coupled }}$ \\
\hline$J / \psi B_{c}^{*}(9451)$ & 1 & 1 & 9451 & 9451 \\
$J / \psi^{8} B_{c}^{* 8}$ & 2 & 2 & 9650 & 9650 \\
{$[c b]^{\mathbf{3}_{\mathrm{c}}}[\bar{c} \bar{c}]^{\mathbf{3}_{\mathrm{c}}}$} & 3 & 3 & 9714 & 9714 \\
$\mathrm{~K}_{1}$ & 4 & 5 & 9695 & \\
& 5 & 6 & 9697 & 9688 \\
$\mathrm{~K}_{2}$ & 6 & 7 & 9613 & \\
& 7 & 8 & 9682 & 9593 \\
$\mathrm{~K}_{3}$ & 8 & 9 & 10184 & \\
& 9 & 10 & 9716 & 9704 \\
$\mathrm{~K}_{4}$ & 10 & 12 & 9717 & 9717 \\
Lowest mass of the fully-coupled result: & & 9451 \\
\hline \hline
\end{tabular}

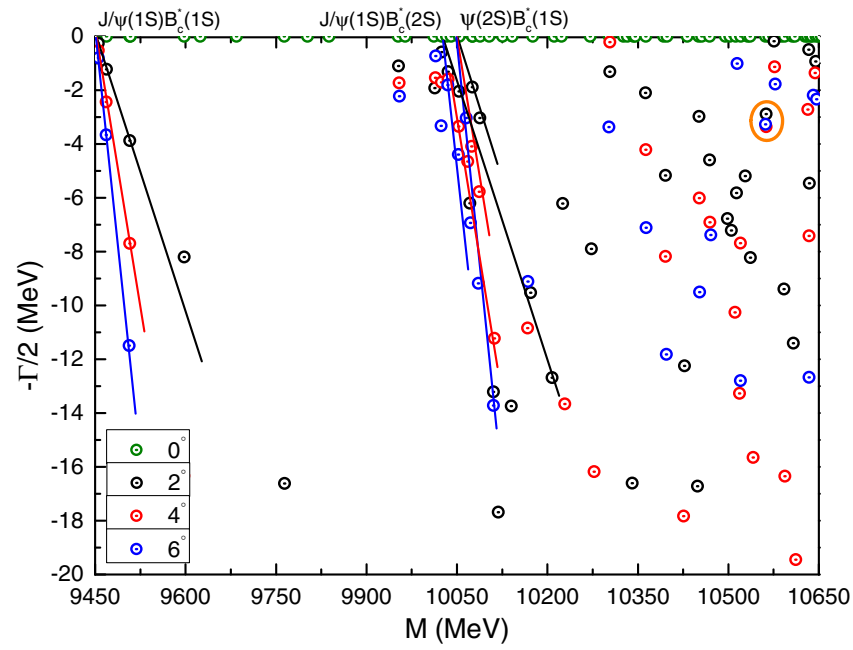

FIG. 10. Complex energy spectrum of the $\bar{c} c \bar{c} b$ system with $J^{P}=2^{+}$from the complete coupled-channel calculation with CSM. The parameter $\theta$ varies from $0^{\circ}$ to $6^{\circ}$. Remember here that, according to the $\mathrm{ABC}$ theorem, (i) a bound state corresponds to a pole on the real axis whose energy is smaller than the mesonmeson threshold and remains unchanged under the complex scaling transformation; (ii) the discretized continuum states are rotated downward by an angle of $2 \theta$ with respect the real axis; and (iii) a resonant state corresponds to a complex pole, that is independent of the angle $\theta$, circled in orange. 
obtained at $10.56 \mathrm{GeV}$, and its width is $6.61 \mathrm{MeV}$. Due to the location in the complex plane, we can identify it as a $\psi(2 S) B_{c}^{*}(1 S)(10563)$ resonance.

\section{G. Charm-bottom system $\bar{b} b \bar{b} c$}

We find some resonance structures with quantum numbers $J^{P}=1^{+}$and $2^{+}$states for the charm-bottom system $\bar{b} b \bar{b} c$. Table XVI summarizes our findings, the details of the calculation are discussed in the following.

\section{The $J^{P}=0^{+}$channel}

There are 20 channels listed in Table XVII. In particular, there are two meson-meson channels, $\eta_{b} \bar{B}_{c}$ and $\Upsilon \bar{B}_{c}^{*}$, two diquark-antidiquark ones, $[c b]^{6 c}[\bar{b} \bar{b}]^{\overline{6} c}$ and $[c b]^{3 c}[\bar{b} \bar{b}]^{\overline{3} c}$, and 14 channels of K-type configurations. Firstly, singlechannel calculations deliver a lowest mass of $15676 \mathrm{MeV}$ for the $\eta_{b} \bar{B}_{c}$, confirming its nature as a scattering state. The color-singlet channel of $\Upsilon \bar{B}_{c}^{*}$ configuration is also a scattering state with a mass of $15812 \mathrm{MeV}$. The remaining excited channels are located within 15.92-16.48 GeV. Although the coupled-channels effect helps in pushing down the lowest masses of each configuration, they are still above the threshold values of $\eta_{b} \bar{B}_{c}$ and $\Upsilon \bar{B}_{c}^{*}$. Moreover, when a complete coupled-bases calculation is performed, the coupling is still too weak to have a bound state.

A complex range analysis on the fully coupled-channels calculation is done and the results are presented in Fig. 11. One can notice that, within a mass region of $15.6-16.5 \mathrm{GeV}$, six continuum states are clearly identified: $\eta_{b}(1 S) \bar{B}_{c}(1 S)$, $\Upsilon(1 S) \bar{B}_{c}^{*}(1 S), \eta_{b}(2 S) \bar{B}_{c}(1 S), \eta_{b}(1 S) \bar{B}_{c}(2 S), \Upsilon(2 S) \bar{B}_{c}^{*}(1 S)$ and $\Upsilon(1 S) \bar{B}_{c}^{*}(2 S)$. However, neither bound nor resonance states are obtained in this case.

\section{The $\mathrm{J}^{P}=1^{+}$channel}

There are 30 channels when considering the $\bar{b} b \bar{b} c$ tetraquark system in $J^{P}=1^{+}$. Table XVIII shows the masses of the color-singlet channels of dimeson structures, $\eta_{b} \bar{B}_{c}^{*}(15750 \mathrm{MeV}), \Upsilon \Upsilon \bar{B}_{c}(15738 \mathrm{MeV})$ and $\Upsilon \bar{B}_{c}^{*}$ $(15812 \mathrm{MeV})$; these results indicate the nature of scattering states. As for the other excited channels, their calculated masses generally locate in the range between $15.95 \mathrm{GeV}$ and $16.44 \mathrm{GeV}$. In the coupled-channels studies of each

TABLE XVI. Predicted resonances of the $\bar{b} b \bar{b} c$ tetraquark system from the complete coupled-channel calculation with CSM. Their masses and widths are summarized in the third and fourth column, respectively, in units of $\mathrm{MeV}$.

\begin{tabular}{lccr}
\hline \hline$J^{P}$ & Resonance & Mass & Width \\
\hline $1^{+}$ & $\Upsilon(1 S) \bar{B}_{c}^{*}(1 S)$ & 16031 & 24.61 \\
& $\Upsilon(2 S) \bar{B}_{c}(1 S)$ & 16303 & 1.80 \\
$2^{+}$ & $\Upsilon(1 S) \bar{B}_{c}^{*}(1 S)$ & 16312 & 1.12 \\
\hline \hline
\end{tabular}

TABLE XVII. Calculation of the $J^{P}=0^{+}$state of the $\bar{b} b \bar{b} c$ system in the real case $\left(\theta=0^{\circ}\right)$. The first and second columns show all possible structures and channels. They are obtained by the necessary bases in spin and color degrees of freedom summarized in the third column. Note also that, in the first column, when corresponding, the theoretical value of the noninteracting meson-meson threshold appears in parentheses. The lowest energies of all channels without considering any channelchannel coupling are listed in the fourth column. The last column shows the lowest energies in a calculation taking into account the couplings among the channels belonging to each structure (configuration). The last row gives the lowest energy from the complete coupled-channel calculation. All results are in units of $\mathrm{MeV}$.

\begin{tabular}{|c|c|c|c|c|}
\hline Structure & Channel & $\xi_{0}^{\alpha} ; \varphi_{\beta}[\alpha ; \beta]$ & $M_{\text {uncoupled }}$ & $M_{\text {coupled }}$ \\
\hline$\eta_{b} \bar{B}_{c}(15676)$ & 1 & {$[1 ; 1]$} & 15676 & \\
\hline$\Upsilon \bar{B}_{c}^{*}(15812)$ & 2 & {$[2 ; 1]$} & 15812 & 15676 \\
\hline$\eta_{b}^{8} \bar{B}_{c}^{8}$ & 3 & {$[1 ; 2]$} & 16091 & \\
\hline$\Upsilon^{8} \bar{B}_{c}^{* 8}$ & 4 & {$[2 ; 2]$} & 16064 & 15976 \\
\hline$[c b]^{\mathbf{6}_{\mathrm{c}}}[\bar{b} \bar{b}]^{\overline{\mathbf{6}}_{\mathrm{c}}}$ & 5 & {$[3 ; 4]$} & 16042 & \\
\hline$[c b]^{\mathbf{3}_{\mathrm{c}}}[\bar{b} \bar{b}]^{\mathbf{3}_{\mathrm{c}}}$ & 6 & {$[4 ; 3]$} & 16058 & 16012 \\
\hline \multirow[t]{4}{*}{$\mathrm{K}_{1}$} & 7 & {$[5 ; 5]$} & 16059 & \\
\hline & 8 & {$[5 ; 6]$} & 15988 & \\
\hline & 9 & {$[6 ; 5]$} & 16089 & \\
\hline & 10 & {$[6 ; 6]$} & 15919 & 15906 \\
\hline \multirow[t]{4}{*}{$\mathrm{K}_{2}$} & 11 & {$[7 ; 7]$} & 16032 & \\
\hline & 12 & {$[7 ; 8]$} & 16059 & \\
\hline & 13 & {$[8 ; 7]$} & 15967 & \\
\hline & 14 & {$[8 ; 8]$} & 16082 & 15892 \\
\hline \multirow[t]{4}{*}{$\mathrm{K}_{3}$} & 15 & [9;9] & 16396 & \\
\hline & 16 & {$[9 ; 10]$} & 16060 & \\
\hline & 17 & {$[10 ; 9]$} & 16037 & \\
\hline & 18 & {$[10 ; 10]$} & 16480 & 15977 \\
\hline \multirow[t]{2}{*}{$\mathrm{K}_{4}$} & 19 & {$[11 ; 12]$} & 16056 & \\
\hline & 20 & {$[12 ; 11]$} & 16048 & 16013 \\
\hline \multicolumn{4}{|c|}{ Lowest mass of the fully-coupled result: } & 15676 \\
\hline
\end{tabular}

configuration, the lowest mass of color singlet dimeson structures remains to be $15738 \mathrm{MeV}$, and the other exotic cases are about $16.0 \mathrm{GeV}$. Meanwhile, last row of Table XVIII indicate that the lowest channel, $\Upsilon \bar{B}_{c}$, is still unbound even in a complete coupled-channels calculation.

In order to find possible resonance states, as those obtained in the previous tetraquark systems, a CSM is employed in the fully coupled-channels computation, and the results are shown in Fig. 12. Within a energy gap of 15.7-16.5 GeV, the three ground states, $\eta_{b} \bar{B}_{c}^{*}, \Upsilon \bar{B}_{c}$ and $\Upsilon \bar{B}_{c}^{*}$, along with their first radial excitations, are well shown in the top panel. Most of the complex-energy dots are well align along the corresponding cut lines, reflecting the nature of scattering states. However, one can notice that there is a stable resonance pole circled by an orange line, the calculated mass and width are $16031 \mathrm{MeV}$ and 


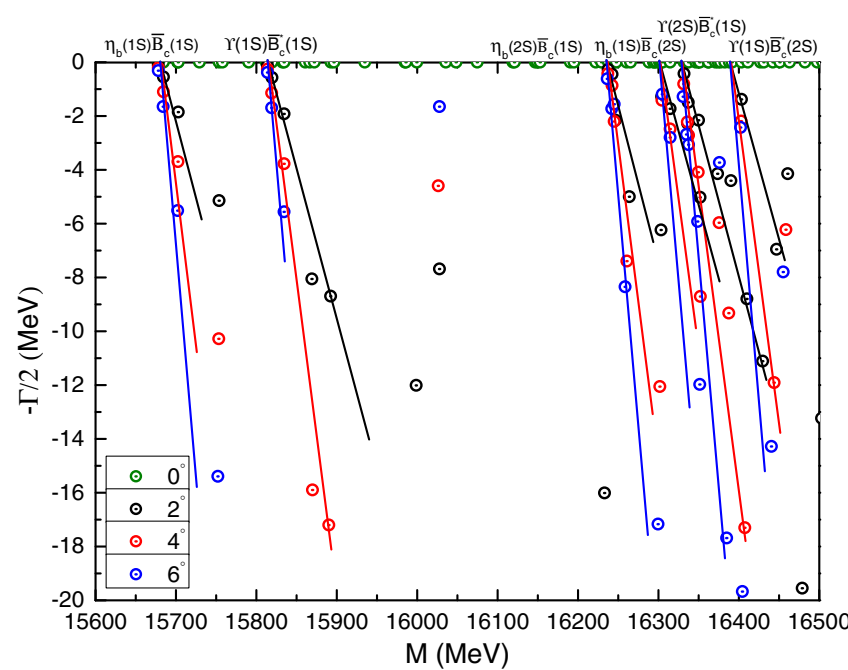

FIG. 11. Complex energy spectrum of the $\bar{b} b \bar{b} c$ system with $J^{P}=0^{+}$from the complete coupled-channel calculation with CSM. The parameter $\theta$ varies from $0^{\circ}$ to $6^{\circ}$. Remember here that, according to the $\mathrm{ABC}$ theorem, (i) a bound state corresponds to a pole on the real axis whose energy is smaller than the mesonmeson threshold and remains unchanged under the complex scaling transformation; (ii) the discretized continuum states are rotated downward by an angle of $2 \theta$ with respect the real axis; and (iii) a resonant state corresponds to a complex pole, that is independent of the angle $\theta$, circled in orange.

$24.61 \mathrm{MeV}$, respectively. It can be identified as a $\Upsilon(1 S) \bar{B}_{c}^{*}(1 S)$ resonance.

In the bottom panel, we further enlarge the energy region between $16.3 \mathrm{GeV}$ and $16.5 \mathrm{GeV}$. One can see that the five radial excited states are clearly shown as scattering states. However, one narrow pole, which is independent of the rotated angle $\theta$, is obtained. Thus, it can be identified as a $\Upsilon(2 S) \bar{B}_{c}(1 S)$ resonance state with a mass and width $16303 \mathrm{MeV}$ and $1.80 \mathrm{MeV}$, respectively.

\section{The $\mathrm{J}^{P}=2^{+}$channel}

Twelve channels are under our investigation in the highest spin state of $\bar{b} b \bar{b} c$ tetraquark system, and the results are listed in Table XIX. First of all, in each single channels calculations, the lowest mass is $15812 \mathrm{MeV}$ for the color-singlet channel of $\Upsilon \bar{B}_{c}^{*}$ state, and this is also the theoretical threshold value. The other excited channels masses are all above $16.0 \mathrm{GeV}$. The fact of unbound state of $\bar{b} b \bar{b} c$ tetraquark system holds for different kinds of coupled-channels calculations.

In additional, Fig. 13 presents the distributions of complex energies of $\bar{b} b \bar{b} c$ tetraquark system in a complete coupledchannels study. In the mass region of $15.8-16.6 \mathrm{GeV}$, three continuum states, $\Upsilon(1 S) \bar{B}_{c}^{*}(1 S), \Upsilon(2 S) \bar{B}_{c}^{*}(1 S)$ and $\Upsilon(1 S) \bar{B}_{c}^{*}(2 S)$, are well shown. However, one narrow resonance pole is obtained and it is quite close to (but below) the threshold of $\Upsilon(2 S) \bar{B}_{c}^{*}(1 S)$. Therefore, it can be identified
TABLE XVIII. Calculation of the $J^{P}=1^{+}$state of the $\bar{b} b \bar{b} c$ system in the real case $\left(\theta=0^{\circ}\right)$. The first and second columns show all possible structures and channels. They are obtained by the necessary bases in spin and color degrees of freedom summarized in the third column. Note also that, in the first column, when corresponding, the theoretical value of the noninteracting meson-meson threshold appears in parentheses. The lowest energies of all channels without considering any channelchannel coupling are listed in the fourth column. The last column shows the lowest energies in a calculation taking into account the couplings among the channels belonging to each structure (configuration). The last row gives the lowest energy from the complete coupled-channel calculation. All results are in units of $\mathrm{MeV}$.

\begin{tabular}{|c|c|c|c|c|}
\hline Structure & Channel & $\xi_{1}^{\alpha} ; \varphi_{\beta}[\alpha ; \beta]$ & $M_{\text {uncoupled }}$ & $M_{\text {coupled }}$ \\
\hline$\eta_{b} \bar{B}_{c}^{*}(15750)$ & 1 & {$[1 ; 1]$} & 15750 & \\
\hline$\Upsilon \bar{B}_{c}(15738)$ & 2 & {$[2 ; 1]$} & 15738 & \\
\hline$\Upsilon \bar{B}_{c}^{*}(15812)$ & 3 & {$[3 ; 1]$} & 15812 & 15738 \\
\hline$\eta_{b}^{8} \bar{B}_{c}^{* 8}$ & 4 & {$[1 ; 2]$} & 16067 & \\
\hline$\Upsilon^{8} \bar{B}_{c}^{8}$ & 5 & {$[2 ; 2]$} & 16070 & \\
\hline$\Upsilon^{8} \bar{B}_{c}^{* 8}$ & 6 & {$[3 ; 2]$} & 16055 & 15993 \\
\hline$[c b]^{\mathbf{6}_{\mathrm{c}}}[\bar{b} \bar{b}]^{\mathbf{6}_{\mathrm{c}}}$ & 7 & {$[4 ; 4]$} & 16032 & \\
\hline$[c b]^{\mathbf{3}_{\mathrm{c}}}[\bar{b} \bar{b}]^{\overline{\mathbf{3}}_{\mathrm{c}}}$ & 8 & {$[5 ; 3]$} & 16049 & \\
\hline$[c b]^{\mathbf{3}_{\mathrm{c}}}[\bar{b} \bar{b}]^{\overline{\mathbf{3}}_{\mathrm{c}}}$ & 9 & {$[6 ; 3]$} & 16068 & 16017 \\
\hline $\mathrm{K}_{1}$ & 10 & {$[7 ; 5]$} & 16013 & \\
\hline & 11 & {$[8 ; 5]$} & 16061 & \\
\hline & 12 & {$[9 ; 5]$} & 16062 & \\
\hline & 13 & {$[7 ; 6]$} & 15990 & \\
\hline & 14 & {$[8 ; 6]$} & 15966 & \\
\hline & 15 & {$[9 ; 6]$} & 15946 & 15922 \\
\hline $\mathrm{K}_{2}$ & 16 & {$[10 ; 7]$} & 16007 & \\
\hline & 17 & {$[11 ; 7]$} & 16039 & \\
\hline & 18 & {$[12 ; 7]$} & 15988 & \\
\hline & 19 & {$[10 ; 8]$} & 16067 & \\
\hline & 20 & {$[11 ; 8]$} & 15993 & \\
\hline & 21 & {$[12 ; 8]$} & 16054 & 15944 \\
\hline $\mathrm{K}_{3}$ & 22 & {$[13 ; 9]$} & 16066 & \\
\hline & 23 & {$[14 ; 9]$} & 16074 & \\
\hline & 24 & {$[15 ; 9]$} & 16442 & \\
\hline & 25 & {$[13 ; 10]$} & 16074 & \\
\hline & 26 & {$[14 ; 10]$} & 16073 & \\
\hline & 27 & {$[15 ; 10]$} & 16051 & 15985 \\
\hline $\mathrm{K}_{4}$ & 28 & {$[16 ; 12]$} & 16053 & \\
\hline & 29 & {$[17 ; 12]$} & 16064 & \\
\hline & 30 & {$[18 ; 11]$} & 16035 & 16018 \\
\hline \multicolumn{4}{|c|}{ Lowest mass of the fully-coupled result: } & 15738 \\
\hline
\end{tabular}

as a $\Upsilon(1 S) \bar{B}_{c}^{*}(1 S)(16312)$ resonance with width is $1.12 \mathrm{MeV}$.

\section{H. Charm-bottom system $\bar{c} b \bar{c} b$}

For the charm-bottom system $\bar{c} b \bar{c} b$, some resonance structures are found with quantum numbers $J^{P(C)}=0^{+(+)}$, 

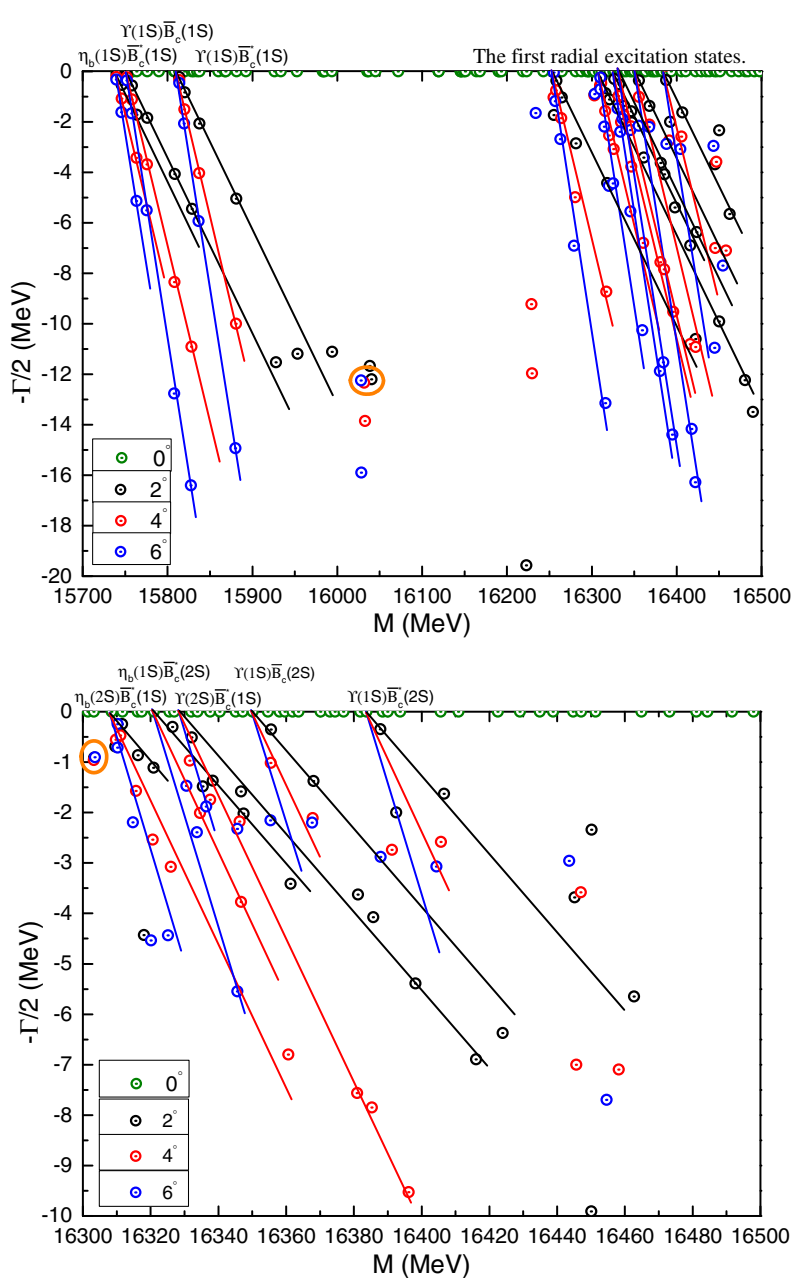

TABLE XIX. Calculation of the $J^{P}=2^{+}$state of the $\bar{b} b \bar{b} c$ system in the real case $\left(\theta=0^{\circ}\right)$. The first and second columns show all possible structures and channels. They are obtained by the necessary bases in spin and color degrees of freedom summarized in the third column. The value in the parenthesis after the first meson-meson structure gives the theoretical result of the noninteracting meson-meson threshold. The lowest energies of all channels without considering any channel-channel coupling are listed in the fourth column. The last column shows the lowest energies in a calculation taking into account the couplings among the channels belonging to each structure (configuration). The last row gives the lowest energy from the complete coupled-channel calculation. All results are in units of $\mathrm{MeV}$.

\begin{tabular}{lcccc}
\hline \hline Structure & Channel & $\xi_{2} ; \varphi_{\beta}[\beta]$ & $M_{\text {uncoupled }}$ & $M_{\text {coupled }}$ \\
\hline$\Upsilon \bar{B}_{c}^{*}(15812)$ & 1 & 1 & 15812 & 15812 \\
$\Upsilon^{8} \bar{B}_{c}^{* 8}$ & 2 & 2 & 16030 & 16030 \\
{$[c b]^{\mathbf{3}_{\mathrm{c}}}[\bar{b} \bar{b}]^{\mathbf{3}_{\mathrm{c}}}$} & 3 & 3 & 16087 & 16087 \\
$\mathrm{~K}_{1}$ & 4 & 5 & 16043 & \\
& 5 & 6 & 16017 & 16008 \\
$\mathrm{~K}_{2}$ & 6 & 7 & 16055 & \\
& 7 & 8 & 16021 & 15980 \\
$\mathrm{~K}_{3}$ & 8 & 9 & 16451 & \\
& 9 & 10 & 16090 & 16065 \\
$\mathrm{~K}_{4}$ & 10 & 12 & 16087 & 16087 \\
\multicolumn{5}{l}{ Lowest mass of the fully-coupled result: } \\
\hline \hline
\end{tabular}

FIG. 12. Top panel: complex energy spectrum of the $\bar{b} b \bar{b} c$ system with $J^{P}=1^{+}$from the complete coupled-channel calculation with CSM. The parameter $\theta$ varies from $0^{\circ}$ to $6^{\circ}$. Bottom panel: enlarged top panel, with real values of energy ranging from $16.30 \mathrm{GeV}$ to $16.50 \mathrm{GeV}$. Remember here that, according to the $\mathrm{ABC}$ theorem, (i) a bound state corresponds to a pole on the real axis whose energy is smaller than the meson-meson threshold and remains unchanged under the complex scaling transformation; (ii) the discretized continuum states are rotated downward by an angle of $2 \theta$ with respect the real axis; and (iii) a resonant state corresponds to a complex pole, that is independent of the angle $\theta$, circled in orange.

$1^{+(-)}$and $2^{+(+)}$. Table XX summarizes the results, and the details are discussed in the following.

\section{The $J^{P(C)}=0^{+(+)}$channel}

There are 18 channels, as listed in Table XXI. The calculated masses of the two dimeson structures in colorsinglet channels are $12550 \mathrm{MeV}$ and $12698 \mathrm{MeV}$, respectively. These are just the theoretical threshold values of di- $B_{c}$ and di- $B_{c}^{*}$, and their relevant hidden-color channels masses are both around $12.91 \mathrm{GeV}$. As for the other exotic structures, diquark-antidiquark and K-types configurations,

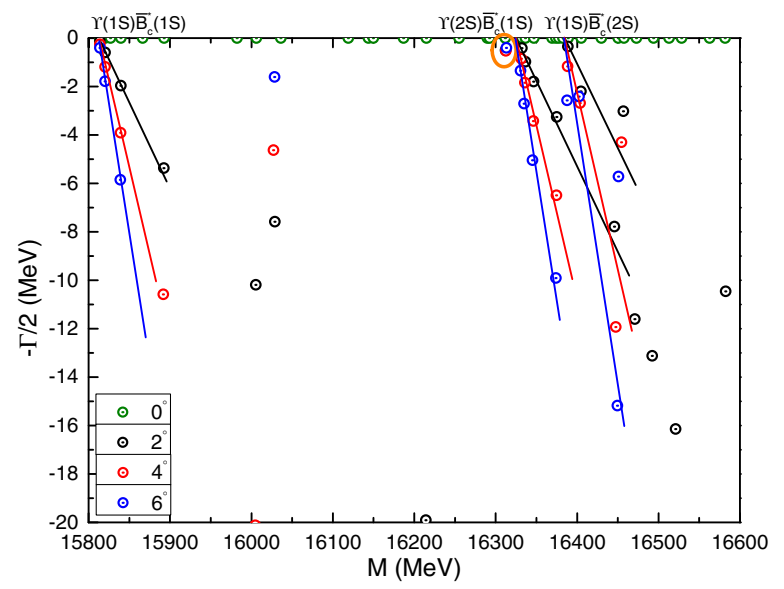

FIG. 13. Complex energy spectrum of the $\bar{b} b \bar{b} c$ system with $J^{P}=2^{+}$from the complete coupled-channel calculation with CSM. The parameter $\theta$ varies from $0^{\circ}$ to $6^{\circ}$. Remember here that, according to the $\mathrm{ABC}$ theorem, (i) a bound state corresponds to a pole on the real axis whose energy is smaller than the mesonmeson threshold and remains unchanged under the complex scaling transformation; (ii) the discretized continuum states are rotated downward by an angle of $2 \theta$ with respect the real axis; and (iii) a resonant state corresponds to a complex pole, that is independent of the angle $\theta$, circled in orange. 
TABLE XX. Predicted resonances of the $\bar{c} b \bar{c} b$ tetraquark system from the complete coupled-channel calculation with CSM. Their masses and widths are summarized in the third and fourth column, respectively, in units of $\mathrm{MeV}$.

\begin{tabular}{lccr}
\hline \hline$J^{P(C)}$ & Resonance & Mass & Width \\
\hline $0^{+(+)}$ & $B_{c}^{*}(1 S) B_{c}^{*}(1 S)$ & 12768 & 1.73 \\
$1^{+(-)}$ & $B_{c}(1 S) B_{c}^{*}(2 S)$ & 13214 & 0.66 \\
$2^{+(+)}$ & $B_{c}^{*}(1 S) B_{c}^{*}(1 S)$ & 12965 & 17.42 \\
& $B_{c}^{*}(1 S) B_{c}^{*}(1 S)$ & 13236 & 4.40 \\
& $B_{c}^{*}(1 S) B_{c}^{*}(1 S)$ & 13248 & 3.41 \\
\hline \hline
\end{tabular}

TABLE XXI. Calculation of the $J^{P(C)}=0^{+(+)}$state of the $\bar{c} b \bar{c} b$ system in the real case $\left(\theta=0^{\circ}\right)$. The first and second columns show all possible structures and channels. They are obtained by the necessary bases in spin and color degrees of freedom summarized in the third column. Note also that, in the first column, when corresponding, the theoretical value of the noninteracting meson-meson threshold appears in parentheses. The lowest energies of all channels without considering any channel-channel coupling are listed in the fourth column. The last column shows the lowest energies in a calculation taking into account the couplings among the channels belonging to each structure (configuration). The last row gives the lowest energy from the complete coupled-channel calculation. All results are in units of $\mathrm{MeV}$.

\begin{tabular}{|c|c|c|c|c|}
\hline Structure & Channel & $\xi_{0}^{\alpha} ; \varphi_{\beta}[\alpha ; \beta]$ & $M_{\text {uncoupled }}$ & $M_{\text {coupled }}$ \\
\hline$B_{c} B_{c}(12550)$ & 1 & {$[1 ; 1]$} & 12550 & \\
\hline$B_{c}^{*} B_{c}^{*}(12698)$ & 2 & {$[2 ; 1]$} & 12698 & 12550 \\
\hline$B_{c}^{8} B_{c}^{8}$ & 3 & {$[1 ; 2]$} & 12949 & \\
\hline$B_{c}^{* 8} B_{c}^{* 8}$ & 4 & {$[2 ; 2]$} & 12910 & 12853 \\
\hline$[b b]^{\mathbf{6}_{\mathrm{c}}}[\bar{c} \bar{c}]^{\mathbf{6}_{\mathrm{c}}}$ & 5 & {$[3 ; 4]$} & 12888 & \\
\hline$[b b]^{\mathbf{3}_{\mathrm{c}}}[\bar{c} \bar{c}]^{\mathbf{3}_{\mathrm{c}}}$ & 6 & {$[4 ; 3]$} & 12867 & 12836 \\
\hline $\mathrm{K}_{1}$ & 7 & {$[5 ; 5]$} & 12833 & \\
\hline & 8 & {$[5 ; 6]$} & 12835 & \\
\hline & 9 & {$[6 ; 5]$} & 12876 & \\
\hline & 10 & {$[6 ; 6]$} & 12742 & 12695 \\
\hline $\mathrm{K}_{2}$ & 11 & {$[7 ; 7]$} & 12806 & \\
\hline & 12 & {$[7 ; 8]$} & 12850 & \\
\hline & 13 & {$[8 ; 7]$} & 12705 & \\
\hline & 14 & {$[8 ; 8]$} & 12889 & 12689 \\
\hline $\mathrm{K}_{3}$ & 15 & {$[9 ; 10]$} & 12868 & \\
\hline & 16 & {$[10 ; 9]$} & 12882 & 12832 \\
\hline $\mathrm{K}_{4}$ & 17 & {$[11 ; 12]$} & 12869 & \\
\hline & 18 & {$[12 ; 11]$} & 12895 & 12839 \\
\hline \multicolumn{4}{|c|}{ Lowest mass of the fully-coupled result: } & 12550 \\
\hline
\end{tabular}

the obtained single-channels masses are generally located in the energy range $12.71-12.89 \mathrm{GeV}$. When the coupledchannels calculations are considered, no bound state is found.

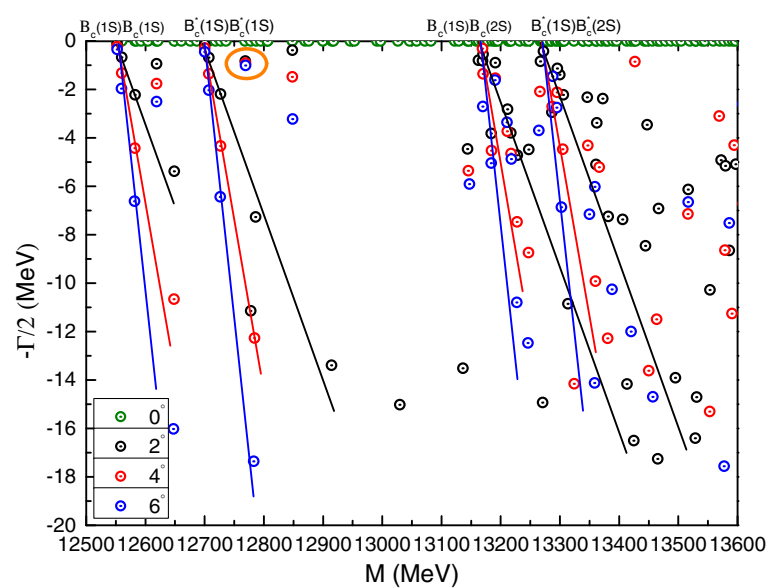

FIG. 14. Complex energy spectrum of the $\bar{c} b \bar{c} b$ system with $J^{P(C)}=0^{+(+)}$from the complete coupled-channel calculation with CSM. The parameter $\theta$ varies from $0^{\circ}$ to $6^{\circ}$. Remember here that, according to the $\mathrm{ABC}$ theorem, (i) a bound state corresponds to a pole on the real axis whose energy is smaller than the mesonmeson threshold and remains unchanged under the complex scaling transformation; (ii) the discretized continuum states are rotated downward by an angle of $2 \theta$ with respect the real axis; and (iii) a resonant state corresponds to a complex pole, that is independent of the angle $\theta$, circled in orange.

Figure 14 shows our results when a complete coupledchannels calculation using the CSM formalism is performed. In an energy region of $12.5-13.6 \mathrm{GeV}$, the scattering states of $B_{c}(1 S) B_{c}(1 S), \quad B_{c}^{*}(1 S) B_{c}^{*}(1 S)$, $B_{c}(1 S) B_{c}(2 S)$, and $B_{c}^{*}(1 S) B_{c}^{*}(2 S)$ are well presented. One stable pole is also found and circled. It is above the $B_{c}^{*}(1 S) B_{c}^{*}(1 S)$ cut lines and thus it can be identified as a di$B_{c}^{*}(1 S)$ resonance with mass and width $12768 \mathrm{MeV}$ and $1.73 \mathrm{MeV}$, respectively.

\section{The $J^{P(C)}=1^{+(-)}$channel}

we have 23 channels as listed in Table XXII. They involve two dimeson configurations, $B_{c} B_{c}^{*}$ and $B_{c}^{*} B_{c}^{*}$ in the singlet- as well as in the hidden-color wave functions, one diquark-antidiquark channel $[b b]^{\mathbf{3}_{\mathrm{c}}}[\bar{c} \bar{c}]^{\overline{3}_{\mathrm{c}}}$, and $18 \mathrm{~K}$-type structures. Firstly, the meson-meson configurations in color-singlet channels are unbound and their calculated masses are $12624 \mathrm{MeV}$ and $12698 \mathrm{MeV}$, respectively. The two hidden-color channels are characterize by a mass of $\sim 12.9 \mathrm{GeV}$, this value is also compatible for the diquark-antidiquark case. The K-type tetraquarks are at $\sim 12.87 \mathrm{GeV}$. Additionally, we do not find any bound state in coupled-channels calculations, which imply couplings in each certain tetraquark configuration and a complete one.

By employing the CSM in a complete coupled-channel investigation, the distributions of eigenenergies are shown in Fig. 15. In the top panel, with an energy range from $12.6 \mathrm{GeV}$ to $13.6 \mathrm{GeV}$, neither bound nor resonance states are found; the scattering states of $B_{c}(1 S) B_{c}^{*}(1 S)$, 
TABLE XXII. Calculation of the $J^{P(C)}=1^{+(-)}$state of the $\bar{c} b \bar{c} b$ system in the real case $\left(\theta=0^{\circ}\right)$. The first and second columns show all possible structures and channels. They are obtained by the necessary bases in spin and color degrees of freedom summarized in the third column. Note also that, in the first column, when corresponding, the theoretical value of the noninteracting meson-meson threshold appears in parentheses. The lowest energies of all channels without considering any channel-channel coupling are listed in the fourth column. The last column shows the lowest energies in a calculation taking into account the couplings among the channels belonging to each structure (configuration). The last row gives the lowest energy from the complete coupled-channel calculation. All results are in units of $\mathrm{MeV}$.

\begin{tabular}{|c|c|c|c|c|}
\hline Structure & Channel & $\xi_{1}^{\alpha} ; \varphi_{\beta}[\alpha ; \beta]$ & $M_{\text {uncoupled }}$ & $M_{\text {coupled }}$ \\
\hline$B_{c} B_{c}^{*}(12624)$ & 1 & {$[1 ; 1]$} & 12624 & \\
\hline$B_{c}^{*} B_{c}^{*}(12698)$ & 2 & {$[3 ; 1]$} & 12698 & 12624 \\
\hline$B_{c}^{8} B_{c}^{* 8}$ & 3 & {$[1 ; 2]$} & 12852 & \\
\hline$B_{c}^{* 8} B_{c}^{* 8}$ & 4 & {$[3 ; 2]$} & 12910 & 12851 \\
\hline$[b b]^{\mathbf{3}_{\mathrm{c}}}[\bar{c} \bar{c}]^{\overline{\mathbf{3}}_{\mathrm{c}}}$ & 5 & {$[6 ; 3]$} & 12878 & 12878 \\
\hline \multirow[t]{6}{*}{$\mathrm{K}_{1}$} & 6 & {$[7 ; 5]$} & 12886 & \\
\hline & 7 & {$[8 ; 5]$} & 12878 & \\
\hline & 8 & {$[9 ; 5]$} & 12863 & \\
\hline & 9 & {$[7 ; 6]$} & 12886 & \\
\hline & 10 & {$[8 ; 6]$} & 12891 & \\
\hline & 11 & {$[9 ; 6]$} & 12855 & 12788 \\
\hline \multirow[t]{6}{*}{$\mathrm{K}_{2}$} & 12 & {$[10 ; 7]$} & 12868 & \\
\hline & 13 & {$[11 ; 7]$} & 12874 & \\
\hline & 14 & {$[12 ; 7]$} & 12826 & \\
\hline & 15 & {$[10 ; 8]$} & 12886 & \\
\hline & 16 & {$[11 ; 8]$} & 12889 & \\
\hline & 17 & {$[12 ; 8]$} & 12875 & 12759 \\
\hline \multirow[t]{3}{*}{$\mathrm{K}_{3}$} & 18 & {$[15 ; 9]$} & 13399 & \\
\hline & 19 & {$[13 ; 10]$} & 12888 & \\
\hline & 20 & {$[14 ; 10]$} & 12887 & 12878 \\
\hline \multirow[t]{3}{*}{$\mathrm{K}_{4}$} & 21 & {$[16 ; 12]$} & 12882 & \\
\hline & 22 & {$[17 ; 12]$} & 12881 & \\
\hline & 23 & {$[18 ; 11]$} & 13336 & 12880 \\
\hline \multicolumn{4}{|c|}{ Lowest mass of the fully-coupled result: } & 12624 \\
\hline
\end{tabular}

$B_{c}^{*}(1 S) B_{c}^{*}(1 S)$ along with their first radial excitation states are however clearly identified.

Nevertheless, when we perform an energy zoom in the bottom panel, apart from the three continuum states of $B_{c}(1 S) B_{c}^{*}(2 S), B_{c}(2 S) B_{c}^{*}(1 S)$, and $B_{c}^{*}(1 S) B_{c}^{*}(2 S)$, a stable pole with mass and width $13214 \mathrm{MeV}$ and $0.66 \mathrm{MeV}$, is found. It is located above the $B_{c}(1 S) B_{c}^{*}(2 S)$ threshold line too.

\section{The $J^{P(C)}=2^{+(+)}$channel:}

For the highest spin state of $\bar{c} b \bar{c} b$ tetraquark system, there are 9 channels under consideration, and the results are listed in Table XXIII. Firstly, in each single-channel
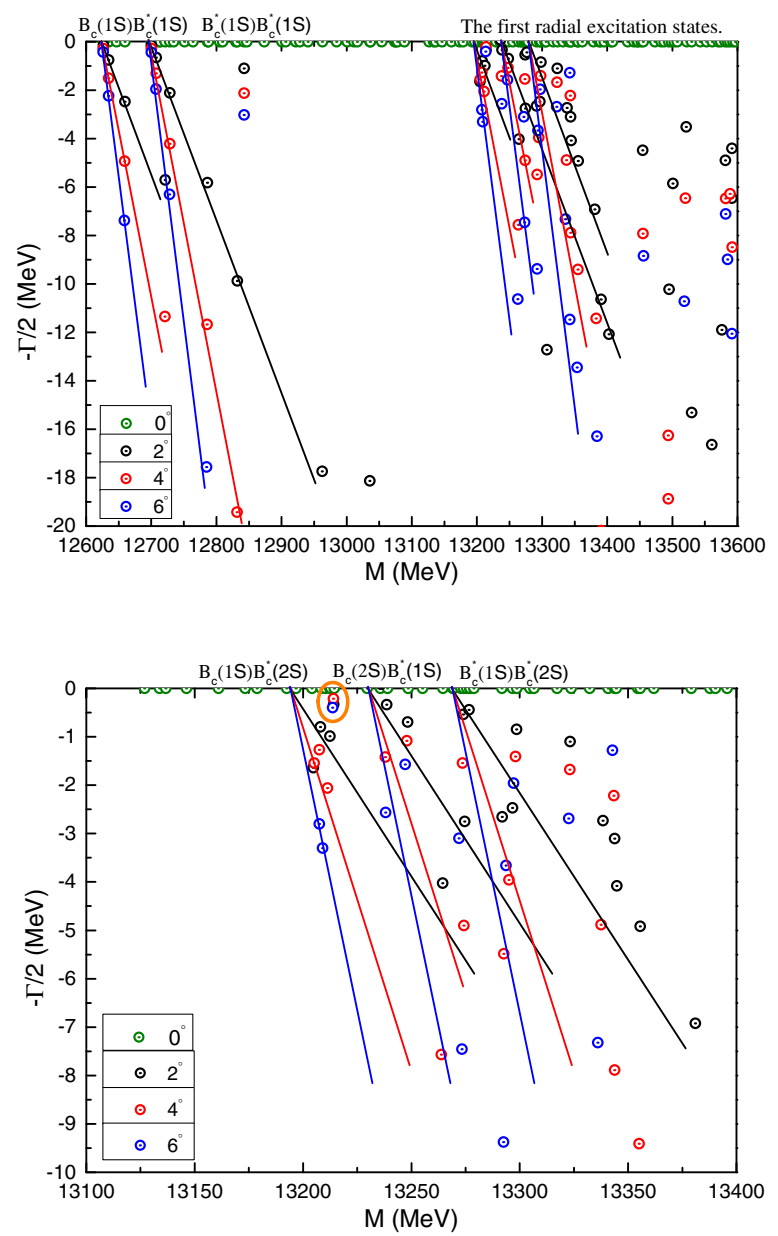

FIG. 15. Top panel: complex energy spectrum of the $\bar{c} b \bar{c} b$ system with $J^{P(C)}=1^{+(-)}$from the complete coupled-channel calculation with CSM. The parameter $\theta$ varies from $0^{\circ}$ to $6^{\circ}$. Bottom panel: enlarged top panel, with real values of energy ranging from $13.10 \mathrm{GeV}$ to $13.40 \mathrm{GeV}$. Remember here that, according to the $\mathrm{ABC}$ theorem, (i) a bound state corresponds to a pole on the real axis whose energy is smaller than the mesonmeson threshold and remains unchanged under the complex scaling transformation; (ii) the discretized continuum states are rotated downward by an angle of $2 \theta$ with respect the real axis; and (iii) a resonant state corresponds to a complex pole, that is independent of the angle $\theta$, circled in orange.

calculation, masses of the color-singlet, hidden-color and diquark-antidiquark structures are $12698 \mathrm{GeV}, 12916 \mathrm{GeV}$ and $12899 \mathrm{GeV}$, respectively. The other $6 \mathrm{~K}$-types masses locate in the range 12.82-12.90 GeV. Obviously, no bound state is found and this fact does not change in the different coupled-channel calculations. The lowest mass, $12698 \mathrm{MeV}$, is just the theoretical threshold value of di- $B_{c}^{*}$.

In a further step, when a complete coupled-channels calculation is performed in the complex range, resonances are obtained and shown in Fig. 16. In a mass region from $12.65-13.65 \mathrm{GeV}$, the scattering states of $B_{c}^{*}(1 S) B_{c}^{*}(1 S)$ and $B_{c}^{*}(1 S) B_{c}^{*}(2 S)$ are well shown. With the rotated angle varying from $0^{\circ}$ to $6^{\circ}$, there are three stable resonance poles 
TABLE XXIII. Calculation of the $J^{P(C)}=2^{+(+)}$state of the $\bar{c} b \bar{c} b$ system in the real case $\left(\theta=0^{\circ}\right)$. The first and second columns show all possible structures and channels. They are obtained by the necessary bases in spin and color degrees of freedom summarized in the third column. Note also that, in the first column, when corresponding, the theoretical value of the noninteracting meson-meson threshold appears in parentheses. The lowest energies of all channels without considering any channel-channel coupling are listed in the fourth column. The last column shows the lowest energies in a calculation taking into account the couplings among the channels belonging to each structure (configuration). The last row gives the lowest energy from the complete coupled-channel calculation. All results are in units of $\mathrm{MeV}$.

\begin{tabular}{lcccc}
\hline \hline Structure & Channel & $\xi_{2} ; \varphi_{\beta}[\beta]$ & $M_{\text {uncoupled }}$ & $M_{\text {coupled }}$ \\
\hline$B_{c}^{*} B_{c}^{*}(12698)$ & 1 & 1 & 12698 & 12698 \\
$B_{c}^{* 8} B_{c}^{* 8}$ & 2 & 2 & 12916 & 12916 \\
{$[b b]^{3_{c}}[\bar{c} \bar{c}]^{\mathbf{3}_{c}}$} & 3 & 3 & 12899 & 12899 \\
$\mathrm{~K}_{1}$ & 4 & 5 & 12834 & \\
& 5 & 6 & 12844 & 12828 \\
$\mathrm{~K}_{2}$ & 6 & 7 & 12819 & \\
& 7 & 8 & 12842 & 12806 \\
$\mathrm{~K}_{3}$ & 8 & 10 & 12900 & 12900 \\
$\mathrm{~K}_{4}$ & 9 & 12 & 12901 & 12901 \\
Lowest mass of the fully-coupled result: & & 12698 \\
\hline \hline
\end{tabular}

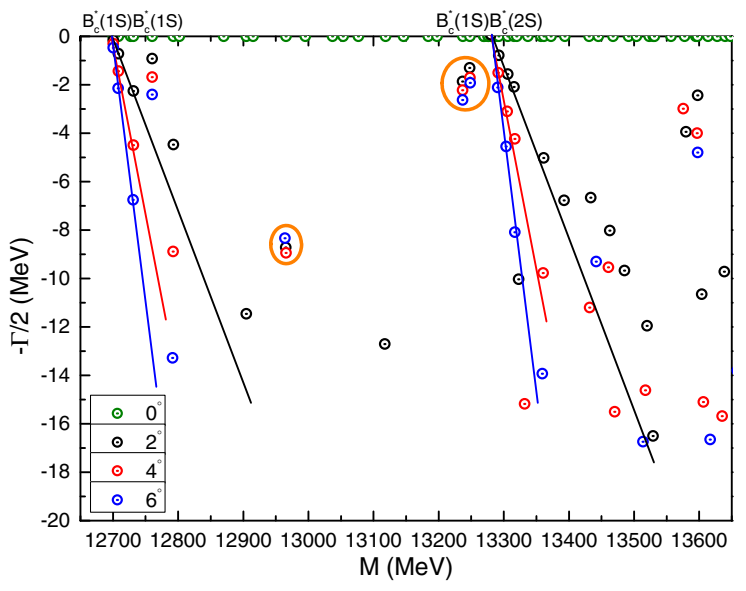

FIG. 16. Complex energy spectrum of the $\bar{c} b \bar{c} b$ system with $J^{P(C)}=2^{+(+)}$from the complete coupled-channel calculation with CSM. The parameter $\theta$ varies from $0^{\circ}$ to $6^{\circ}$. Remember here that, according to the $\mathrm{ABC}$ theorem, (i) a bound state corresponds to a pole on the real axis whose energy is smaller than the mesonmeson threshold and remains unchanged under the complex scaling transformation; (ii) the discretized continuum states are rotated downward by an angle of $2 \theta$ with respect the real axis; and (iii) a resonant state corresponds to a complex pole, that is independent of the angle $\theta$, circled in orange. in the complex plane and they are marked with circles. The lowest one, whose mass and width are $12965 \mathrm{MeV}$ and $17.42 \mathrm{MeV}$, can be identified as a $B_{c}^{*}(1 S) B_{c}^{*}(1 S)$ resonance. For the other two poles, which are quite close to each other, the calculated masses and widths are $(13236 \mathrm{MeV}$,

TABLE XXIV. Predicted resonances of the $\bar{c} c \bar{b} b$ tetraquark system from the complete coupled-channel calculation with CSM. Their masses and widths are summarized in the third and fourth column, respectively, in units of $\mathrm{MeV}$.

\begin{tabular}{lrcc}
\hline \hline$J^{P(C)}$ & Resonance & Mass & Width \\
\hline $0^{+(+)}$ & $J / \psi(1 S) \Upsilon(1 S)$ & 12820 & 0.62 \\
& $\psi(2 S) \Upsilon(1 S)$ & 13449 & 2.81 \\
$1^{+( \pm)}$ & $J / \psi(1 S) \Upsilon(1 S)$ & 12858 & 0.60 \\
& $\eta_{c}(1 S) \Upsilon(2 S)$ & 13002 & 0.94 \\
& $\psi(2 S) \Upsilon(1 S)$ & 13290 & 1.81 \\
$2^{+(+)}$ & $J / \psi(1 S) \Upsilon(1 S)$ & 12826 & 1.25 \\
& $\psi(2 S) \Upsilon(1 S)$ & 13321 & 3.40 \\
\hline \hline
\end{tabular}

TABLE XXV. Calculation of the $J^{P(C)}=0^{+(+)}$state of the $\bar{c} c \bar{b} b$ system in the real case $\left(\theta=0^{\circ}\right)$. The first and second columns show all possible structures and channels. They are obtained by the necessary bases in spin and color degrees of freedom summarized in the third column. Note also that, in the first column, when corresponding, the theoretical value of the noninteracting mesonmeson threshold appears in parentheses. The lowest energies of all channels without considering any channel-channel coupling are listed in the fourth column. The last column shows the lowest energies in a calculation taking into account the couplings among the channels belonging to each structure (configuration). The last row gives the lowest energy from the complete coupled-channel calculation. All results are in units of $\mathrm{MeV}$.

\begin{tabular}{|c|c|c|c|c|}
\hline Structure & Channel & $\xi_{0}^{\alpha} ; \varphi_{\beta}[\alpha ; \beta]$ & $M_{\text {uncoupled }}$ & $M_{\text {coupled }}$ \\
\hline$\eta_{c} \eta_{b}(12369)$ & 1 & {$[1 ; 1]$} & 12369 & \\
\hline$J / \psi \Upsilon(12565)$ & 2 & {$[2 ; 1]$} & 12565 & 12369 \\
\hline$\eta_{c}^{8} \eta_{b}^{8}$ & 3 & {$[1 ; 2]$} & 12858 & \\
\hline$J / \psi^{8} \Upsilon^{8}$ & 4 & {$[2 ; 2]$} & 12838 & 12809 \\
\hline$[c b]^{\mathbf{3}_{\mathrm{c}}}[\bar{c} \bar{b}]^{\overline{\mathbf{3}}_{\mathrm{c}}}$ & 5 & {$[3 ; 3]$} & 12861 & \\
\hline$[c b]^{\mathbf{6}_{\mathrm{c}}}[\bar{c} \bar{b}]^{\mathbf{6}_{\mathrm{c}}}$ & 6 & {$[3 ; 4]$} & 12868 & \\
\hline 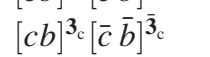 & 7 & {$[4 ; 3]$} & 12892 & \\
\hline$[c b]^{\mathbf{6}_{\mathrm{c}}}[\bar{c} \bar{b}]^{\mathbf{6}_{\mathrm{c}}}$ & 8 & {$[4 ; 4]$} & 12784 & 12707 \\
\hline $\mathrm{K}_{1}$ & 9 & {$[5 ; 5]$} & 12854 & \\
\hline & 10 & {$[5 ; 6]$} & 12771 & \\
\hline & 11 & {$[6 ; 5]$} & 12872 & \\
\hline & 12 & {$[6 ; 6]$} & 12601 & 12599 \\
\hline $\mathrm{K}_{3}$ & 13 & {$[9 ; 9]$} & 12786 & \\
\hline & 14 & {$[9 ; 10]$} & 12896 & \\
\hline & 15 & {$[10 ; 9]$} & 12876 & \\
\hline & 16 & {$[10 ; 10]$} & 12872 & 12717 \\
\hline \multicolumn{4}{|c|}{ Lowest mass of the fully-coupled result: } & 12369 \\
\hline
\end{tabular}


4.40 MeV) and (13248 MeV, $3.41 \mathrm{MeV})$, respectively. Their dominant channel is again $B_{c}^{*}(1 S) B_{c}^{*}(1 S)$.

\section{Charm-bottom system $\bar{c} c \bar{b} b$}

For the charm-bottom system $\bar{c} c \bar{b} b$, some resonance structures are found with quantum numbers $J^{P(C)}=0^{+(+)}$, $1^{+( \pm)}$and $2^{+(+)}$. Table XXIV summarizes our results and the details of the computation can be found in the following. Note herein that the $K_{1}$-type structure (Fig. 1(c)) is equivalent to the $K_{2}$-type one (Fig. 1(d)) for the $\bar{c} c \bar{b} b$ system, and this identity also holds for $K_{3^{-}}$ (Fig. 1(e)) and $K_{4}$-type (Fig. 1(f)) configurations. Hence, only $K_{1}$ - and $K_{3}$-types are employed in the following tables without losing any generality.

\section{The $J^{P(C)}=0^{+(+)}$channel}

There are 16 channels listed in Table XXV. In particular, the $\eta_{c} \eta_{b}$ and $J / \psi \Upsilon$ dimeson channels have masses at $12369 \mathrm{MeV}$ and $12565 \mathrm{MeV}$, and so they are both scattering states. Their corresponding hidden-color channels are almost degenerate with masses close to $12.85 \mathrm{GeV}$. The diquark-antidiquark and K-type configurations are all located in the energy range $12.77-12.89 \mathrm{GeV}$, except the $K_{1}$ channel with a mass at $12.60 \mathrm{GeV}$. No bound state is available in any of the three possible calculations: singlechannel, configuration-type channels calculation, and fullchannel computation.

Let us now focus on Fig. 17, which presents the distribution of complex eigenenergies of $J^{P(C)}=0^{+(+)} \bar{c} c \bar{b} b$

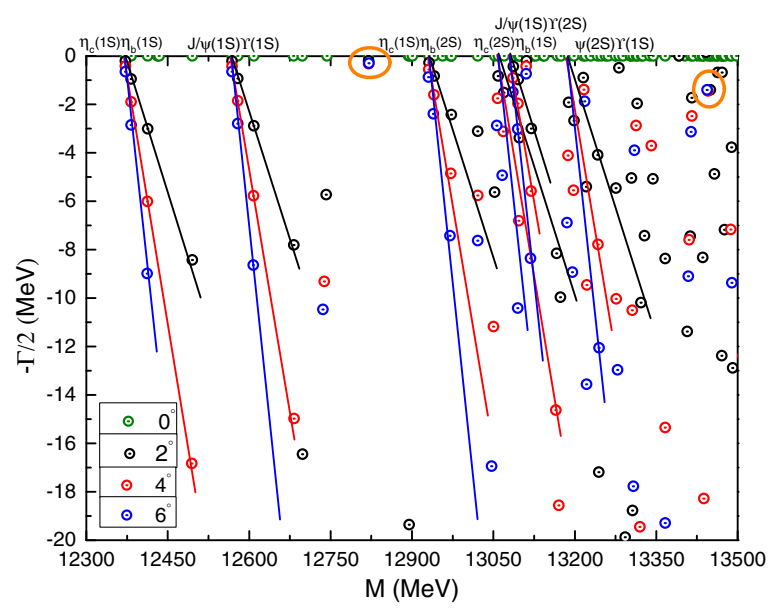

FIG. 17. Complex energy spectrum of the $\bar{c} c \bar{b} b$ system with $J^{P(C)}=0^{+(+)}$from the complete coupled-channel calculation with CSM. The parameter $\theta$ varies from $0^{\circ}$ to $6^{\circ}$. Remember here that, according to the $\mathrm{ABC}$ theorem, (i) a bound state corresponds to a pole on the real axis whose energy is smaller than the mesonmeson threshold and remains unchanged under the complex scaling transformation; (ii) the discretized continuum states are rotated downward by an angle of $2 \theta$ with respect the real axis; and (iii) a resonant state corresponds to a complex pole, that is independent of the angle $\theta$, circled in orange. tetraquarks in a complete coupled-channels calculation by $\mathrm{CSM}$, in a mass region $12.3-13.5 \mathrm{GeV}$. The scattering nature of $\eta_{c}(1 S) \eta_{b}(1 S), J / \psi(1 S) \Upsilon(1 S), \eta_{c}(1 S) \eta_{b}(2 S)$, $\eta_{c}(2 S) \eta_{b}(1 S), J / \psi(1 S) \Upsilon(2 S)$, and $\psi(2 S) \Upsilon(1 S)$ is well established. However, there are two resonance poles which do not depend on the variation of the rotated angle $\theta$. One pole is identified as a $J / \psi(1 S) \Upsilon(1 S)(12820)$ narrow resonance with $\Gamma=0.62 \mathrm{MeV}$, another is $\psi(2 S) \Upsilon(1 S)(13449)$ with $\Gamma=2.81 \mathrm{MeV}$.

\section{The $J^{P(C)}=1^{+( \pm)}$channel}

Table XXVI shows the 22 channels available in this case. The three meson-meson structures of $\eta_{c} \Upsilon, J / \psi \eta_{b}$ and $J / \psi \Upsilon$ in color-singlet channels are all unbound, and their

TABLE XXVI. Calculation of the $J^{P(C)}=1^{+( \pm)}$state of the $\bar{c} c \bar{b} b$ system in the real case $\left(\theta=0^{\circ}\right)$. The first and second columns show all possible structures and channels. They are obtained by the necessary bases in spin and color degrees of freedom summarized in the third column. Note also that, in the first column, when corresponding, the theoretical value of the noninteracting meson-meson threshold appears in parentheses. The lowest energies of all channels without considering any channel-channel coupling are listed in the fourth column. The last column shows the lowest energies in a calculation taking into account the couplings among the channels belonging to each structure (configuration). The last row gives the lowest energy from the complete coupled-channel calculation. All results are in units of $\mathrm{MeV}$.

\begin{tabular}{|c|c|c|c|c|}
\hline Structure & Channel & $\xi_{1}^{\alpha} ; \varphi_{\beta}[\alpha ; \beta]$ & $M_{\text {uncoupled }}$ & $M_{\text {coupled }}$ \\
\hline$\eta_{c} \Upsilon(12431)$ & 1 & {$[1 ; 1]$} & 12431 & \\
\hline$J / \psi \eta_{b}(12503)$ & 2 & {$[2 ; 1]$} & 12503 & \\
\hline$J / \psi \Upsilon(12565)$ & 3 & {$[3 ; 1]$} & 12565 & 12431 \\
\hline$\eta_{c}^{8} \Upsilon^{8}$ & 4 & {$[1 ; 2]$} & 12861 & \\
\hline$J / \psi^{8} \eta_{b}^{8}$ & 5 & {$[2 ; 2]$} & 12867 & \\
\hline$J / \psi^{8} \Upsilon^{8}$ & 6 & {$[3 ; 2]$} & 12855 & 12843 \\
\hline$[c b]^{\mathbf{3}_{\mathrm{c}}}[\bar{c} \bar{b}]^{\mathbf{3}_{\mathrm{c}}}$ & 7 & {$[4 ; 3]$} & 12888 & \\
\hline$[c b]^{\mathbf{3}_{\mathrm{c}}}[\bar{c} \bar{b}]^{\mathbf{3}_{\mathrm{c}}}$ & 8 & {$[6 ; 3]$} & 12903 & \\
\hline$[c b]^{\mathbf{6}_{\mathrm{c}}}[\bar{c} \bar{b}]^{\mathbf{6}_{\mathrm{c}}}$ & 9 & {$[4 ; 4]$} & 12858 & \\
\hline$[c b]^{\mathbf{6}_{\mathrm{c}}}[\bar{c} \bar{b}]^{\mathbf{6}_{\mathrm{c}}}$ & 10 & {$[6 ; 4]$} & 12817 & 12769 \\
\hline $\mathrm{K}_{1}$ & 11 & {$[7 ; 5]$} & 12879 & \\
\hline & 12 & {$[8 ; 5]$} & 12872 & \\
\hline & 13 & {$[9 ; 5]$} & 12876 & \\
\hline & 14 & {$[7 ; 6]$} & 12748 & \\
\hline & 15 & {$[8 ; 6]$} & 12759 & \\
\hline & 16 & {$[9 ; 6]$} & 12636 & 12635 \\
\hline $\mathrm{K}_{3}$ & 17 & {$[13 ; 9]$} & 12863 & \\
\hline & 18 & {$[14 ; 9]$} & 12824 & \\
\hline & 19 & [15;9] & 12864 & \\
\hline & 20 & {$[13 ; 10]$} & 12907 & \\
\hline & 21 & {$[14 ; 10]$} & 12901 & \\
\hline & 22 & {$[15 ; 10]$} & 12894 & 12768 \\
\hline \multicolumn{4}{|c|}{ Lowest mass of the fully-ce } & 12431 \\
\hline
\end{tabular}


calculated masses are $12431 \mathrm{MeV}, 12503 \mathrm{MeV}$, and $12565 \mathrm{MeV}$, respectively. Their hidden-color channels are around $12.86 \mathrm{GeV}$. As for the other excited channels in diquark-antidiquark and K-type structures, they lie in the energy range 12.75-12.90 GeV. The coupled-masses of each certain configurations are all above $12.6 \mathrm{GeV}$, and hence no bound state is found.

Nevertheless, three narrow resonances are obtained in a complete coupled-channels calculation by CSM, as shown in Fig. 18. They are $J / \psi(1 S) \Upsilon(1 S)(12858)$, $\eta_{c}(1 S) \Upsilon(2 S)(13002)$, and $\psi(2 S) \Upsilon(1 S)(13290)$, with two-body strong decay widths $0.60 \mathrm{MeV}, 0.94 \mathrm{MeV}$ and
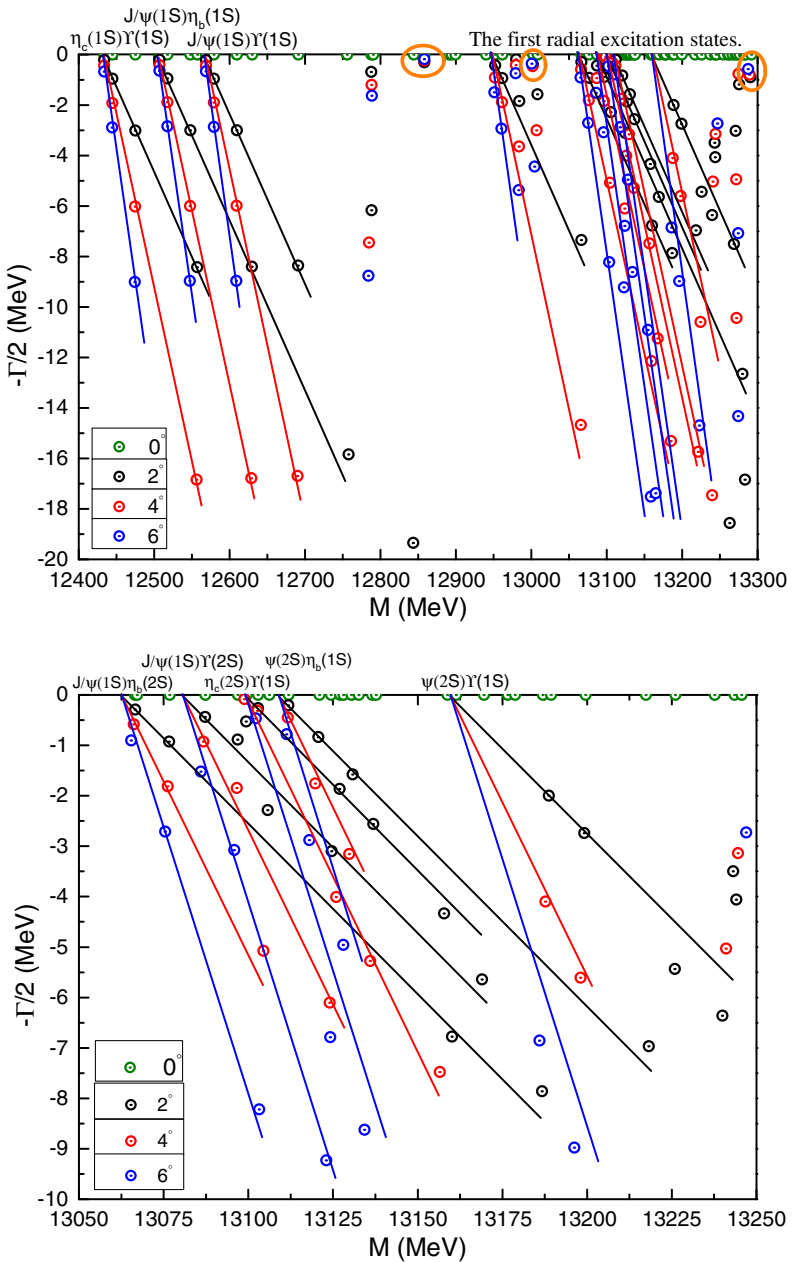

FIG. 18. Top panel: complex energy spectrum of the $\bar{c} c \bar{b} b$ system with $J^{P(C)}=1^{+( \pm)}$from the complete coupled-channel calculation with CSM. The parameter $\theta$ varies from $0^{\circ}$ to $6^{\circ}$. Bottom panel: enlarged top panel, with real values of energy ranging from $13.05 \mathrm{GeV}$ to $13.25 \mathrm{GeV}$. Remember here that, according to the $\mathrm{ABC}$ theorem, (i) a bound state corresponds to a pole on the real axis whose energy is smaller than the mesonmeson threshold and remains unchanged under the complex scaling transformation; (ii) the discretized continuum states are rotated downward by an angle of $2 \theta$ with respect the real axis; and (iii) a resonant state corresponds to a complex pole, that is independent of the angle $\theta$, circled in orange.
TABLE XXVII. Calculation of the $J^{P(C)}=2^{+(+)}$state of the $\bar{c} c \bar{b} b$ system in the real case $\left(\theta=0^{\circ}\right)$. The first and second columns show all possible structures and channels. They are obtained by the necessary bases in spin and color degrees of freedom summarized in the third column. Note also that, in the first column, when corresponding, the theoretical value of the noninteracting mesonmeson threshold appears in parentheses. The lowest energies of all channels without considering any channel-channel coupling are listed in the fourth column. The last column shows the lowest energies in a calculation taking into account the couplings among the channels belonging to each structure (configuration). The last row gives the lowest energy from the complete coupled-channel calculation. All results are in units of $\mathrm{MeV}$.

\begin{tabular}{|c|c|c|c|c|}
\hline Structure & Channel & $\xi_{2} ; \varphi_{\beta}[\beta]$ & $M_{\text {uncoupled }}$ & $M_{\text {coupled }}$ \\
\hline$J / \psi \Upsilon(12565)$ & 1 & 1 & 12565 & 12565 \\
\hline$J / \psi^{8} \Upsilon^{8}$ & 2 & 2 & 12885 & 12885 \\
\hline$\left.[c b]^{\mathbf{3}_{\mathrm{c}}[\bar{c}} \bar{b}\right]^{\overline{\mathbf{3}}_{\mathrm{c}}}$ & 3 & 3 & 12926 & \\
\hline$[c b]^{\mathbf{6}_{\mathrm{c}}}[\bar{c} \bar{b}]^{\mathbf{6}_{\mathrm{c}}}$ & 4 & 4 & 12880 & 12830 \\
\hline \multirow[t]{2}{*}{$\mathrm{K}_{1}$} & 5 & 5 & 12900 & \\
\hline & 6 & 6 & 12771 & 12771 \\
\hline \multirow[t]{2}{*}{$\mathrm{K}_{3}$} & 7 & 9 & 12887 & \\
\hline & 8 & 10 & 12933 & 12844 \\
\hline \multicolumn{4}{|c|}{ Lowest mass of the fully-coupled result: } & 12565 \\
\hline
\end{tabular}

$1.81 \mathrm{MeV}$, respectively. Additionally, an energy zoom shown in the bottom panel of Fig. 18 gives a more clear picture on the scattering nature for $J / \psi(1 S) \eta_{b}(2 S)$, $J / \psi(1 S) \Upsilon(2 S), \quad \eta_{c}(2 S) \Upsilon(1 S), \quad \psi(2 S) \eta_{b}(1 S) \quad$ and $\psi(2 S) \Upsilon(1 S)$ and the fact that no resonance state is found.

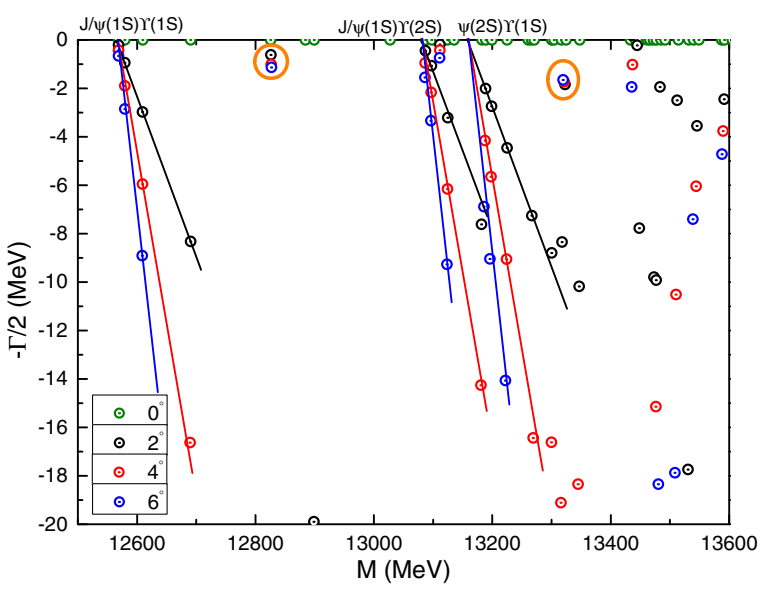

FIG. 19. Complex energy spectrum of the $\bar{c} c \bar{b} b$ system with $J^{P(C)}=2^{+(+)}$from the complete coupled-channel calculation with CSM. The parameter $\theta$ varies from $0^{\circ}$ to $6^{\circ}$. Remember here that, according to the $\mathrm{ABC}$ theorem, (i) a bound state corresponds to a pole on the real axis whose energy is smaller than the mesonmeson threshold and remains unchanged under the complex scaling transformation; (ii) the discretized continuum states are rotated downward by an angle of $2 \theta$ with respect the real axis; and (iii) a resonant state corresponds to a complex pole, that is independent of the angle $\theta$, circled in orange. 


\section{The $J^{P(C)}=3^{+(+)}$channel}

8 channels characterize this case and they are listed in Table XXVII: one meson-meson structure, $J / \psi \Upsilon$, two diquark-antidiquark structures, $[c b]^{\mathbf{3}_{\mathrm{c}}}[\bar{c} \bar{c}]^{\overline{3}_{\mathrm{c}}}$ and $[c b]^{\mathbf{6}_{c}}[\bar{c} \bar{b}]^{\mathbf{6}_{c}}$, and four K-type configurations. The lowest computed mass, $12565 \mathrm{MeV}$, is at the theoretical threshold of $J / \psi \Upsilon$. The other excited channels locate within 12.77$12.93 \mathrm{GeV}$. Meanwhile, the coupled-channels effect is still too weak to form a bound state.

However, two resonances are obtained in a fully coupled-channels investigation by CSM. In particular, Fig. 19 shows the distributions of scattering states of $J / \psi(1 S) \Upsilon(1 S), \quad J / \psi(1 S) \Upsilon(2 S)$, and $\psi(2 S) \Upsilon(1 S)$. Moreover, there are two stable poles marked with orange circles. One is identified as a $J / \psi(1 S) \Upsilon(1 S)(12826)$ resonance, and the other as a $\psi(2 S) \Upsilon(1 S)(13321)$ state, their widths are $1.25 \mathrm{MeV}$ and $3.40 \mathrm{MeV}$, respectively.

\section{SUMMARY}

The fully-heavy tetraquark system $Q Q \bar{Q} \bar{Q}(Q=c, b)$ in its $S$-wave state, viz. the $c c \bar{c} \bar{c}, b b \bar{b} \bar{b}, c b \bar{c} \bar{c}, c b \bar{b} \bar{b}, b b \bar{c} \bar{c}$, and $c b \bar{c} \bar{b}$ tetraquark lowest-lying states with quantum numbers $J^{P(C)}=0^{+(+)}, 1^{+( \pm)}$, and $2^{+(+)}$, have been systemically investigated by using a potential model which mimics the lattice-QCD findings about the interaction between a heavy quark and antiquark pair. That is to say, the quark model involves the so-called Cornell potential supplemented by a spin-spin dependent interaction. Furthermore, the four-body problem is solved by a Gaussian expansion method where each relative motion is expanded by a Gaussian basis whose sizes are in geometric progression. The complex scaling method is used to identify bound, resonance and scattering states in the complex energy plane. And all four-body configurations, including meson-meson, diquark-antidiquark, and K-type structures, as well as the couplings among themselves, are comprehensively considered.

Several narrow resonances have been determined in any of the all-heavy tetraquark systems investigated. Tables IV, VIII, XII, XVI, XX, and XXIV summarize our findings. Amongst all the results presented, the following are of particular interest: (i) For the narrow di- $J / \psi$ structure at $6.9 \mathrm{GeV}$ recently seen by the LHCb collaboration, our investigation indicates that four resonances are consistent: $J / \psi(1 S) \psi(2 S)(6875)$ and $J / \psi(1 S) \psi(2 S)(6987)$ with $J^{P(C)}=0^{+(+)}, \quad \eta_{c}(2 S) J / \psi(1 S)(6885)$ with $J^{P(C)}=1^{+(-)}$, and $J / \psi(1 S) \psi(2 S)(7007)$ with $J^{P(C)}=2^{+(+)}$.

(ii) Within the invariant-mass range $6.2-6.8 \mathrm{GeV}$ where a broad structure was reported by the $\mathrm{LHCb}$ collaboration, our study provides four possible candidates. They are the resonances $\eta_{c}(1 S) \eta_{c}(2 S)(6640)$ and $\eta_{c}(1 S) \eta_{c}(2 S)(6762)$ with $J^{P(C)}=0^{+(+)}$and the resonances $J / \psi(1 S) J / \psi(1 S)(6274)$ and $J / \psi(1 S) J / \psi(1 S)(6653)$ with $J^{P(C)}=1^{+(-)}$.

(iii) Related with the possible fully-heavy tetraquark structure around $7.2 \mathrm{GeV}$ also reported by the $\mathrm{LHCb}$ collaboration, our investigation provides a possible candidate with mass $7195 \mathrm{MeV}$ and width $8 \mathrm{MeV}$, in the $J / \psi(1 S) \psi(2 S)$ channel and with quantum numbers $J^{P(C)}=0^{+(+)}$.

The predicted resonances of the fully-heavy tetraquark systems can be investigated in future experiments such as the ATLAS, CMS, and LHCb at CERN. In particular, the CMS experiment has momentum and mass resolutions comparable in size with those of the LHCb; however, the CMS collaboration has already collected 20 times more data than the $\mathrm{LHCb}$ one. From a theoretical point of view, the fully-heavy tetraquark systems can be safely studied from a nonrelativistic approximation and thus they constitute a nice platform to examine and improve the phenomenological potential model applied to multiquark systems.

\section{ACKNOWLEDGMENTS}

G. Yang would like to thank J. Zhao, L. He and Q. Wang for constructive discussions. Work supported by: National Natural Science Foundation of China under Grants No. 11535005 and No. 11775118; Ministerio Español de Ciencia e Innovación under Grant no. PID2019107844 GB-C22; and Junta de Andalucía, Contract Nos. P18-FR-5057 and Operativo FEDER Andalucía 2014-2020 UHU-1264517.
[1] R. Aaij et al. (LHCb Collaboration), Phys. Rev. Lett. 122, 222001 (2019).

[2] R. Aaij et al. (LHCb Collaboration), Phys. Rev. Lett. 115, 072001 (2015).

[3] S. K. Choi et al. (Belle Collaboration), Phys. Rev. Lett. 91, 262001 (2003).
[4] B. Aubert et al. (BABAR Collaboration), Phys. Rev. Lett. 95, 142001 (2005).

[5] M. Ablikim et al. (BESIII Collaboration), Phys. Rev. Lett. 110, 252001 (2013).

[6] V. Khachatryan et al. (CMS Collaboration), J. High Energy Phys. 05 (2017) 013. 
[7] S. Durgut, Ph.D. thesis at University of Iowa, 2018, https://ir .uiowa.edu/etd/6411/.

[8] S. Durgut, APS April Meeting 2018, http://meetings.aps .org/Meeting/APR18/Session/U09.6.

[9] K. Yi, Int. J. Mod. Phys. A 33, 1850224 (2018).

[10] L. C. Bland et al. ( $A_{N} \mathrm{DY}$ Collaboration), arXiv: 1909.03124.

[11] R. Aaij et al. (LHCb Collaboration), J. High Energy Phys. 10 (2018) 086.

[12] The CMS Collaboration, Phys. Lett. B 808, 135578 (2020).

[13] R. Aaij et al. (LHCb Collaboration), Sci. Bull. 65, 1983 (2020).

[14] K. Yi, Int. J. Mod. Phys. A 28, 1330020 (2013).

[15] J. Richard, Sci. Bull. 65, 1954 (2020), arXiv:2008.01962.

[16] K. Chao and S. Zhu, Sci. Bull. 65, 1952 (2020).

[17] A. V. Berezhnoy, A. V. Luchinsky, and A. A. Novoselov, Phys. Rev. D 86, 034004 (2012).

[18] A. Esposito and A. D. Polosa, Eur. Phys. J. C 78, 782 (2018).

[19] M. N. Anwar, J. Ferretti, F.-K. Guo, E. Santopinto, and B.-S. Zou, Eur. Phys. J. C 78, 647 (2018).

[20] M. A. Bedolla, J. Ferretti, C. D. Roberts, and E. Santopinto, Eur. Phys. J. C 80, 1004 (2020).

[21] Z.-G. Wang, Eur. Phys. J. C 77, 432 (2017).

[22] W. Chen, H.-X. Chen, X. Liu, T. G. Steele, and S.-L. Zhu, Phys. Lett. B 773, 247 (2017).

[23] Y. Bai, S. Lu, and J. Osborne, Phys. Lett. B 798, 134930 (2019).

[24] C. Becchi, A. Giachino, L. Maiani, and E. Santopinto, Phys. Lett. B 806, 135495 (2020).

[25] V. R. Debastiani and F. S. Navarra, Chin. Phys. C 43, 013105 (2019).

[26] A. V. Berezhnoy, A. K. Likhoded, A. V. Luchinsky, and A. A. Novoselov, Phys. Rev. D 84, 094023 (2011).

[27] M. Karliner, S. Nussinov, and J. L. Rosner, Phys. Rev. D 95, 034011 (2017).

[28] W. Heupel, G. Eichmann, and C. S. Fischer, Phys. Lett. B 718, 545 (2012).

[29] J.-M. Richard, A. Valcarce, and J. Vijande, Phys. Rev. D 95, 054019 (2017).

[30] J. Wu, Y.-R. Liu, K. Chen, X. Liu, and S.-L. Zhu, Phys. Rev. D 97, 094015 (2018).

[31] X. Chen, Eur. Phys. J. A 55, 106 (2019).

[32] M.-S. Liu, Qi-F. Lü, X.-H. Zhong, and Q. Zhao, Phys. Rev. D 100, 016006 (2019).

[33] G.-J. Wang, L. Meng, and S.-L. Zhu, Phys. Rev. D 100, 096013 (2019).

[34] J.-M. Richard, A. Valcarce, and J. Vijande, Phys. Rev. C 97, 035211 (2018).

[35] C. Hughes, E. Eichten, and C. T. H. Davies, Phys. Rev. D 97, 054505 (2018).

[36] P. Lundhammar and T. Ohlsson, Phys. Rev. D 102, 054018 (2020).

[37] M. S. Liu, F. X. Liu, X. H. Zhong, and Q. Zhao, arXiv: 2006.11952.

[38] J. F. Giron and R. F. Lebed, Phys. Rev. D 102, 074003 (2020).

[39] Z. G. Wang, Chin. Phys. C 44, 113106 (2020).

[40] M. Karliner and J. L. Rosner, Phys. Rev. D 102, 114039 (2020).
[41] Q. Lü, D. Chen, and T. Dong, Eur. Phys. J. C 80, 871 (2020).

[42] R. M. Albuquerque, S. Narison, A. Rabemananjara, D. Rabetiarivony, and G. Randriamanatrika, Phys. Rev. D 102, 094001 (2020).

[43] J. Sonnenschein and D. Weissman, Eur. Phys. J. C 81, 25 (2021).

[44] X. Jin, Y. Xue, H. Huang, and J. Ping, Eur. Phys. J. C 80, 1083 (2020).

[45] R. N. Faustov and V. O. Galkin, Phys. Rev. D 102, 114030 (2020).

[46] J. Zhao, S. Shi, and P. Zhuang, Phys. Rev. D 102, 114001 (2020).

[47] M. C. Gordillo, F. De Soto, and J. Segovia, Phys. Rev. D 102, 114007 (2020).

[48] X. Dong, V. Baru, F. Guo, C. Hanhart, and A. Nefediev, Phys. Rev. Lett. 126, 132001 (2021).

[49] Y. Ma and H. Zhang, arXiv:2009.08376.

[50] H. Chen, W. Chen, X. Liu, and S. Zhu, Sci. Bull. 65, 1994 (2020).

[51] X. Wang, Q. Lin, H. Xu, Y. Xie, Y. Huang, and X. Chen, Phys. Rev. D 102, 116014 (2020).

[52] J. Wang, D. Chen, X. Liu, and T. Matsuki, Phys. Rev. D 103, 071503 (2021).

[53] F. Feng, Y. Huang, Y. Jia, W. Sang, X. Xiong, and J. Zhang, arXiv:2009.08450.

[54] G. Yang, J. Ping, and J. Segovia, Symmetry 12, 1869 (2020).

[55] T. Kawanai and S. Sasaki, Phys. Rev. D 85, 091503 (2012).

[56] E. Hiyama, Y. Kino, and M. Kamimura, Prog. Part. Nucl. Phys. 51, 223 (2003).

[57] J. Aguilar and J. M. Combes, Commun. Math. Phys. 22, 269 (1971).

[58] E. Balslev and J. M. Combes, Commun. Math. Phys. 22, 280 (1971).

[59] B. Simon, Commun. Math. Phys. 27, 1 (1972).

[60] Y. K. Ho, Phys. Rep. 99, 1 (1983).

[61] N. Moiseyev, Phys. Rep. 302, 212 (1998).

[62] S. Aoyama, T. Myo, K. Kato, and K. Ikeda, Prog. Theor. Phys. 116, 1 (2006).

[63] T. Myo, Y. Kikuchi, H. Masui, and K. Kato, Prog. Part. Nucl. Phys. 79, 1 (2014).

[64] E. Hiyama, M. Isaka, M. Kamimura, T. Myo, and T. Motoba, Phys. Rev. C 91, 054316 (2015).

[65] E. Hiyama, R. Lazauskas, J. Carbonell, and M. Kamimura, Phys. Rev. C 93, 044004 (2016).

[66] G. Yang, J. Ping, and J. Segovia, Phys. Rev. D 101, 014001 (2020).

[67] G. Yang, J. Ping, and J. Segovia, Phys. Rev. D 102, 054023 (2020).

[68] G. Yang, J. Ping, and J. Segovia, Phys. Rev. D 101, 074030 (2020).

[69] G. Yang, J. Ping, and J. Segovia, Phys. Rev. D 103, 074011 (2021).

[70] J. Zhao, K. Zhou, S. Chen, and P. Zhuang, Prog. Part. Nucl. Phys. 114, 103801 (2020).

[71] M. Harvey, Nucl. Phys. A352, 326 (1981).

[72] J. Vijande, A. Valcarce, and N. Barnea, Phys. Rev. D 79, 074010 (2009).

[73] G. Yang and J. Ping, Phys. Rev. D 95, 014010 (2017). 